\title{
Exploring novel herbicidin analogues by transcriptional regulator overexpression and MS/MS molecular networking
}

\author{
Yuanyuan Shi ${ }^{1,2}$, Renjie $\mathrm{Gu}^{1}$, Yihong $\mathrm{Li}^{2}$, Xinwei Wang ${ }^{2}$, Weicong Ren ${ }^{1}$, Xingxing $\mathrm{Li}^{1,2}$, Lifei Wang ${ }^{1,2}$, Yunying Xie ${ }^{2^{*}}$ \\ and Bin Hong ${ }^{1,2^{*}}$ (D)
}

\begin{abstract}
Background: Herbicidin F has an undecose tricyclic furano-pyrano-pyran structure with post-decorations. It was detected from Streptomyces mobaraensis US-43 fermentation broth as a trace component by HPLC-MS analysis. As herbicidins exhibit herbicidal, antibacterial, antifungal and antiparasitic activities, we are attracted to explore more analogues for further development.
\end{abstract}

Results: The genome of S. mobaraensis US-43 was sequenced and a herbicidin biosynthetic gene cluster ( $h c d$ ) was localized. The cluster contains structural genes, one transporter and three potential transcription regulatory genes. Overexpression of the three regulators respectively showed that only hcdR2 overexpression significantly improved the production of herbicidin F, and obviously increased the transcripts of 7 structural genes as well as the transporter gene. After performing homology searches using BLASTP in the GenBank database, 14 hcd-like clusters were found with a cluster-situated $h c d R 2$ homologue. These HcdR2 orthologues showed overall structural similarity, especially in the C-terminal DNA binding domain. Based on bioinformatics analysis, a 21-bp consensus binding motif of HcdR2 was detected within 30 promoter regions in these genome-mined clusters. EMSA results verified that HcdR2 bound to the predicted consensus sequence. Additionally, we employed molecular networking to explore novel herbicidin analogues in $h c d R 2$ overexpression strain. As a result, ten herbicidin analogues including six new compounds were identified based on MS/MS fragments. Herbicidin O was further purified and confirmed by ${ }^{1} \mathrm{H}$ NMR spectrum.

Conclusions: A herbicidin biosynthetic gene cluster ( $h c d$ ) was identified in S. mobaraensis US-43. HcdR2, a member of LuxR family, was identified as the pathway-specific positive regulator, and the production of herbicidin F was dramatically increased by overexpression of $h c d R 2$. Combined with molecular networking, ten herbicidin congeners including six novel herbicidin analogues were picked out from the secondary metabolites of $h c d R 2$ overexpression strain. The orthologues of herbicidin F pathway-specific regulator HcdR2 were present in most of the genome-mined homologous biosynthetic gene clusters, which possessed at least one consensus binding motif with LuxR family characteristic. These results indicated that the combination of overexpression of $h c d R 2$ orthologous regulator and molecular networking might be an effective way to exploit the "cryptic" herbicidin-related biosynthetic gene clusters for discovery of novel herbicidin analogues.

Keywords: Regulator hcdR2, Molecular networking, Herbicidin analogues

\footnotetext{
*Correspondence: xieyy@imb.pumc.edu.cn; binhong69@hotmail.com; hongbin@imb.pumc.edu.cn

${ }^{1} \mathrm{NHC}$ Key Laboratory of Biotechnology of Antibiotics, Institute of Medicinal Biotechnology, Chinese Academy of Medical Sciences \& Peking Union Medical College, No.1 Tiantan Xili, Beijing 100050, China ${ }^{2}$ CAMS Key Laboratory of Synthetic Biology for Drug Innovation, Institute of Medicinal Biotechnology, Chinese Academy of Medical Sciences \& Peking Union Medical College, No.1 Tiantan Xili, Beijing 100050, China
} 


\section{Background}

Streptomyces mobaraensis US-43 (former named S. verticillus var. pingyangensis $\mathrm{n}$. var, CPCC 203575), isolated from a soil sample collected from Pingyang, Zhejiang Province, China, produced a series of glycopeptide antibiotics such as bleomycin analogues [1-3]. Among them, pingyangmycin and boanmycin have been approved by SFDA for cancer treatment in China. As a preserved strain in our laboratory, its secondary metabolites in different fermentation conditions were analyzed to explore new compounds, and piericidin A1 and a group of isocoumarins have been obtained (Additional file 1: Figure S1). Additionally, a trace component was detected by LC-MS and speculated as herbicidin F (1) based on UV spectrum and MS/MS fragmentation profile. Herbicidins (Fig. 1) are adenosine-derived nucleoside antibiotics that have a characteristic tricyclic furano-pyrano-pyran structure with different decorations. They have been isolated from S. saganonensis [4-7], S. sp. L-9-10 [8], S. scopuliridis RB72 [9] and S. sp. CB01388 [10]. Herbicidins show selective herbicidal activity toward dicotyledonous plants [4], and also exhibit antialgal [7] and antifungal [6] activities. Recently, a report highlights the herbicidin scaffold for anti-Cryptosporidium drug development [10]. The complex chemical structures and diverse biological activities have attracted our attention for further exploration of herbicidin congeners and their biosynthesis mechanisms. Although the structure of herbicidin $\mathrm{F}$ was reported [6], there were no reports about its biosynthetic gene cluster at the beginning of this work. But at that time a Chinese patent by Tang's group [11] has demonstrated the minimal gene cluster of aureonuclemycin (Fig. 1), the bare tricyclic core structure of herbicidins, produced by $S$. aureus var. suzhoueusis. It contains four necessary genes $(a n m B, a n m C, a n m D$ and $a n m E)$ by inframe deletion and heterologous expression, which is reported in a recent paper that elucidated the herbicidin tailoring pathway [12]. Therefore, the four genes responsible for tricyclic core assembly provided important clues to identify herbicidin biosynthetic gene cluster. Here we report the successful mapping and identification of a herbicidin biosynthetic gene cluster $(h c d)$ in $S$. mobaraensis US-43 by bioinformatics analysis, which is largely homologous to recently reported her in S. sp. L-9-10 [13] and $h b c$ in $S$. sp. KIB-027 [12] responsible for herbicidin biosynthesis. Furthermore, the pathway-specific regulator a<smiles></smiles>

Aureonuclemycin

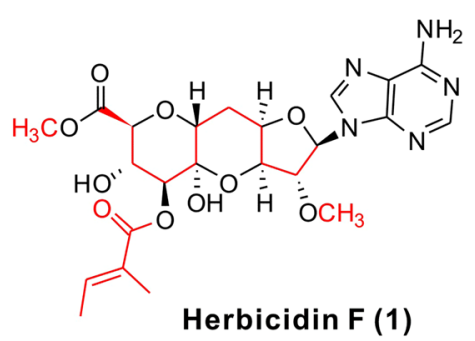

Herbicidin F (1)

\section{b}

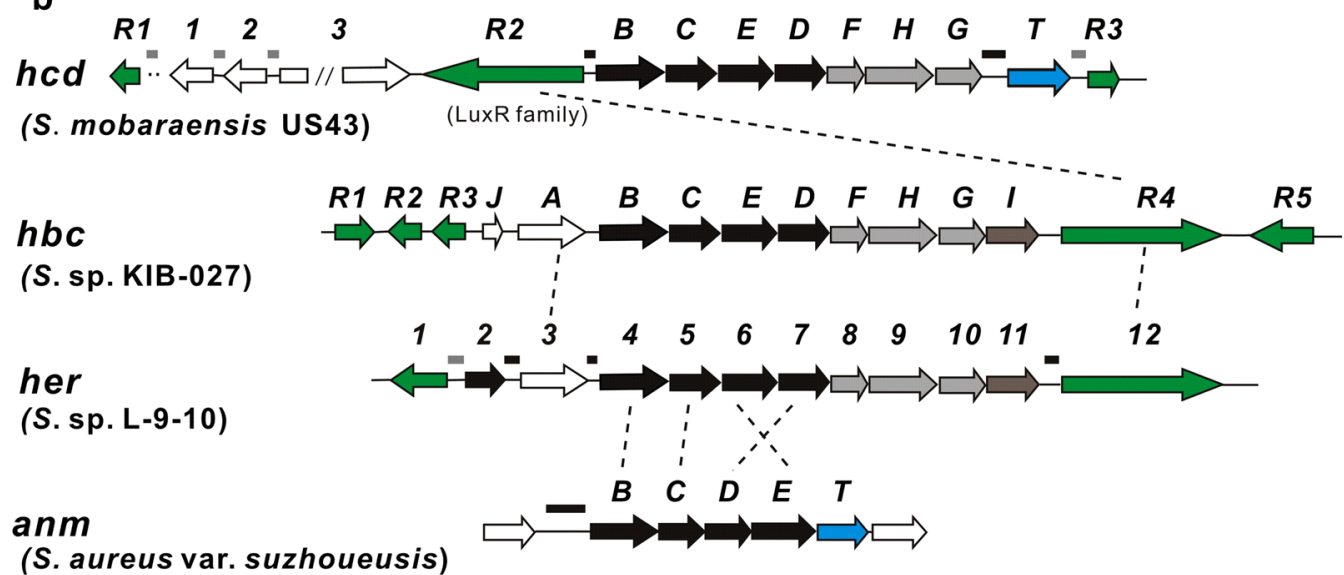

(S. aureus var. suzhoueusis)

Fig. 1 Chemical structures and biosynthetic gene clusters of aureonuclemycin and herbicidin F. a The structures of aureonuclemycin and herbicidin F (1). b Organization of the hcd, hbc, her and anm biosynthetic gene clusters. Lines above the clusters are intergenic regions for FIMO analysis. Black lines contain sequence matching with the consensus binding motif and gray lines don't have the matched sequence 
was identified by overexpression of the potential regulators located near the $h c d$ cluster, and $h c d R 2$ exerted a significant positive role in the production of herbicidin F, which leads to the acquisition of enough amount of herbicidin $\mathrm{F}$ for structural determination by NMR spectra.

After significant improvement of the production of herbicidin F, molecular networking, a computational strategy that assists organization of the tandem MS/MS data [14], was used to identify novel herbicidin congeners in $h c d R 2$ overexpression strain. Based on the assumption that related structures can produce similar fragment patterns in tandem mass spectra, molecular networking produce an MS/MS spectral similarity map that allows the visualization of structurally related molecules [15], which makes the identification of analogues more efficiently. The workflow of molecular networking, available on the Global Natural Product Social Molecular Networking (GNPS, https://gnps.ucsd.edu/ProteoSAFe/static/gnpssplash.jsp) web site, has been successfully applied in the discovery of novel natural products [16-19]. In our work, after LC-MS/MS analysis of the secondary metabolites of the pathway-specific positive regulator overexpression strain, we employed GNPS for further exploration of herbicidin analogues. Six new herbicidins were firstly reported here by MS/MS analysis. Among them, herbicidin $\mathrm{O}$ was purified and further confirmed by proton nuclear magnetic resonance ( ${ }^{1} \mathrm{H}$ NMR) spectrum.

\section{Results}

Bioinformatics identification of the herbicidin biosynthesis gene cluster ( $h c d)$ in S. mobaraensis US-43

Streptomyces mobaraensis US-43, a well-studied strain in our lab, was previously identified to produce a series of bleomycin analogues. Through LC-MS analysis of its fermentation broth, some trace components were detected and a compound (1) with UV spectrum and MS/MS fragmentation profile identical to herbicidin $\mathrm{F}$ attracted our attention. Among the reported herbicidins, herbicidin $\mathrm{F}$ showed better inhibitory activity against Trychophyton mentagrophytes, T. rubrum, T. asteroids, T. megninii and some other fungi and markedly non-toxic to animals $[4,6]$. As aureonuclemycin was identified to bear the bare nucleoside core of herbicidin F (Fig. 1a), its necessary biosynthesis genes $(a n m B, a n m C$, anmD and anmE) [11, 12] (Fig. 1b) were used as targets to explore the possible herbicidin gene cluster. The draft genome of S. mobaraensis US-43 was sequenced (GenBank Accession No. VOKX00000000, Additional file 1: Figure S1). By BLASTP and antiSMASH analysis of the genome of S. mobaraensis US-43 (Additional file 1: Table S1), there was only one predicted cluster containing four correspondingly homologous genes in one operon (Fig. 1b). Downstream of the four core genes, there are two methyltransferase genes which were speculated to be involved in the methylation of herbicidin F. Upstream, there's one $\beta$-ketoacyl synthase that was first thought might be responsible for the biosynthesis of tiglyl moiety in herbicidin F. One transporter, three transcriptional regulators and some other genes were located nearby. Thus we hypothesized that this gene cluster, named $h c d$ cluster, is potentially responsible for the herbicidin $\mathrm{F}$ biosynthetic pathway. The organization of hcd cluster is shown in Fig. $1 \mathrm{~b}$ and the proposed function of each ORF is given in Table 1. During the preparation of this manuscript, the herbicidin biosynthetic gene clusters in $S$. sp. L-9-10 [13] and S. sp. KIB-027 [12] were reported in succession

Table 1 Annotation and predicted function of genes in hcd cluster

\begin{tabular}{|c|c|c|c|c|}
\hline Gene & Name & Size (aa) & Identities & BLAST annotation \\
\hline smo5214 & $\operatorname{hcdR3}$ & 261 & & Transcriptional regulator, SARP family \\
\hline smo5216 & hcdT & 503 & & Major facilitator transporter \\
\hline smo5217 & $h c d G$ & 367 & her10 (68.83\%) & O-Methyltransferase \\
\hline smo5218 & $h c d H$ & 540 & Her9 (685.54\%) & Beta-lactamase \\
\hline smo5219 & $h c d F$ & 286 & $\operatorname{Her} 8(63.50 \%)$ & Methyltransferase \\
\hline smo5220 & $h c d D$ & 370 & anmD (65.26\%)/her7 (74.93\%) & Oxidoreductase \\
\hline smo5221 & hcdE & 435 & anmE (55.30\%)/her6 (71.56\%) & Radical SAM domain protein \\
\hline smo5222 & hcdC & 372 & anmC (52.82\%)/her5 (56.23\%) & Oxidoreductase/dehydrogenase \\
\hline smo5223 & $h c d B$ & 558 & $a n m B(56.24 \%) / h e r 4$ (64.43\%) & S-Adenosine-L-homocysteine hydrolase \\
\hline smo5225 & hcdR2 & 930 & her12 (30.43\%) & LuxR family transcriptional regulator \\
\hline smo5226 & hcd3 & 2613 & & Beta-ketoacyl synthase \\
\hline smo5227 & hcd2 & 348 & & Polyprenyl synthetase \\
\hline smo5228 & hcdl & 359 & & $\begin{array}{l}\text { Terpene synthase/cyclase metal-bind- } \\
\text { ing domain protein }\end{array}$ \\
\hline smo5236 & hcdR1 & 227 & & SARP family transcriptional regulator \\
\hline
\end{tabular}


(Fig. 1b). In our cluster, $h c d B \sim H$ is homologous to both her4 $\sim 10$ and $h b c B \sim H$. Two genes, hbcl/her11 encoding a cytochrome $\mathrm{P} 450$ monooxygenase and $h b c A$ / her3 encoding a ketoacyl-ACP synthase III (KAS III) were absent in the $h c d$ cluster. The transporter HcdT is of major facilitator superfamily which is absent in her and $h b c$ cluster (Fig. 1b). There are three transcriptional regulators situated nearby, and among them, $h c d R 1$ and $h c d R 3$ encode regulators belong to SARP (Streptomyces antibiotic regulatory protein) family while HcdR2 (with its homologues in her and $h b c$ clusters) is classified in LuxR family (Table 1).

\section{Identification of the pathway-specific regulator of $h c d$ cluster}

As three possible regulators were identified near the predicted $h c d$ cluster, we firstly constructed the overexpression strains of each regulator and detected the influence on the production of herbicidin F (1) to determine whether the predicted gene cluster was responsible for the biosynthesis of herbicidin $\mathrm{F}$ and which one was its pathway-specific regulator. The coding region of each $h c d R$ was cloned into the pSET152 [20]-derived expression plasmid pL646 [21] (containing a $\phi C 31$ attP-int locus) under the control of a strong constitutive promoter ermEp*. The resulting plasmids were respectively introduced into the wild type strain $S$. mobaraensis US-43 by conjugation to give the overexpression strains designated as US43/pL-hcdR1, US43/pL-hcdR2 and US43/ pL-hcdR3. The plasmid pSET152 was also transferred to get strain US43/pSET152 as a control. These strains were fermented simultaneously and each of the cultivated broth was analyzed by HPLC. The results showed that only in the strain of US43/pL-hcdR2, the production level of compound $\mathbf{1}$ was significantly increased by about 20-fold (Fig. 2a), which made the separation and purification of 1 much easier. The strain US43/pL-hcdR2 fermentations were scaled up and $11 \mathrm{mg}$ of compound $\mathbf{1}$ was obtained. Then it was confirmed as herbicidin $\mathrm{F}$ by MS/MS fragments (Additional file 1: Figure S2) and comparison with the reported NMR spectra [8] (Additional file 1: Table S2).

As herbicidin F was structurally determined, the significant increase of herbicidin $\mathrm{F}$ production in $h c d R 2$ overexpression strain suggested that transcription regulator HcdR2 played a positive role in herbicidin F biosynthesis. The gene expression analysis was conducted using quantitative RT-PCR to examine the involvement of the 3 regulatory genes in transcription regulation of genes in $h c d$ cluster. The relative level of the transcripts of genes within the cluster were analyzed together with regulators at about $48 \mathrm{~h}$. Compared to control strain US43/ pSET152, the transcripts of $h c d B \sim T$ and $h c d R 2$ were obviously increased in US43/pL-hcdR2, while the transcripts of $h c d 1, h c d 2$ and $h c d 3$ were almost unchanged (Fig. 2b). In US43/pL-hcdR1 and US43/pL-hcdR3, when the overexpressed regulator was upregulated as expected, the transcripts of the above genes have no obvious change compared with that in US43/pSET152 (Fig. 2c, d). These results were consistent with the production level of hebicidin $\mathrm{F}$ and confirmed that $h c d R 2$ is the pathwayspecific positive regulator for the biosynthesis of herbicidin F. What is more, the transcript analysis of US43/ pL-hcdR2 suggested that the operon $h c d B \sim H$ and $h c d T$ is responsible for its biosynthesis. In contrast to our previous prediction of the involvement of $\mathrm{Hcd} 3$ (a $\beta$-ketoacyl synthase) in the biosynthesis of tiglyl moiety, the transcript analysis results suggested that none of $h c d 1 \sim 3$ was involved in the biosynthesis of herbicidin F.

\section{HcdR2 is a conserved pathway-specific regulator for herbicidin production}

HcdR2 belongs to LuxR family and contains a typical helix-turn-helix (HTH) DNA binding domain (DBD) at the $\mathrm{C}$-terminus. Interestingly, BLASTP analysis in GenBank using $h c d B / C / D / E$ as a query showed the existence of 15 more $h c d / h b c / h e r / a n m-l i k e$ clusters in actinomycetes (Fig. 3). The majority of them (11 clusters) also have a cluster-situated transcriptional regulator belonging to LuxR family. These regulators, together with Her12, show $30-43 \%$ amino acid identity to HcdR2 over the full length of the protein except the regulator in S. mobaraensis NBRC 13819 (99.78\% amino acid identity). HHpred and BLASTP analyses of these regulators show high 3D structure similarities with conserved domains including an N-terminal AAA ATPase domain and a C-terminal helix-turn-helix (HTH) DNA binding domain (DBD). Furthermore, the alignment of DBD domains of these orthologues of HcdR2 with the closest DBD structural neighbor of LuxR family regulator TraR shows overall homology and displays the domain architecture of a tetrahelical version of the HTH motif (Fig. 4a) using on-line ESPript sever [22] (mean similarity 66.74\%). The HTH architecture was responsible for multipolarity and binding specific DNA sites near target promoters to modulate gene expression. Therefore we took each $h c d B \mathrm{p}$ and other intergenic regions in total 17 clusters (14 mined-clusters plus $h c d$, her and anm, marked in Figs. 1 and 3, which had 48 sequences in total), except for $h b c$ in $S$. sp. KIB027 (sequence is not available in GenBank) and cluster in Kitasatospora sp. MBT63 (sequence of the cluster is not complete), to search the possible HcdR2 consensus binding sequence using the on-line program MEME Suite 5.0.4 [23-25].

Firstly, a possible HcdR2 consensus binding sequence with highest score was identified in 13 promoter regions 

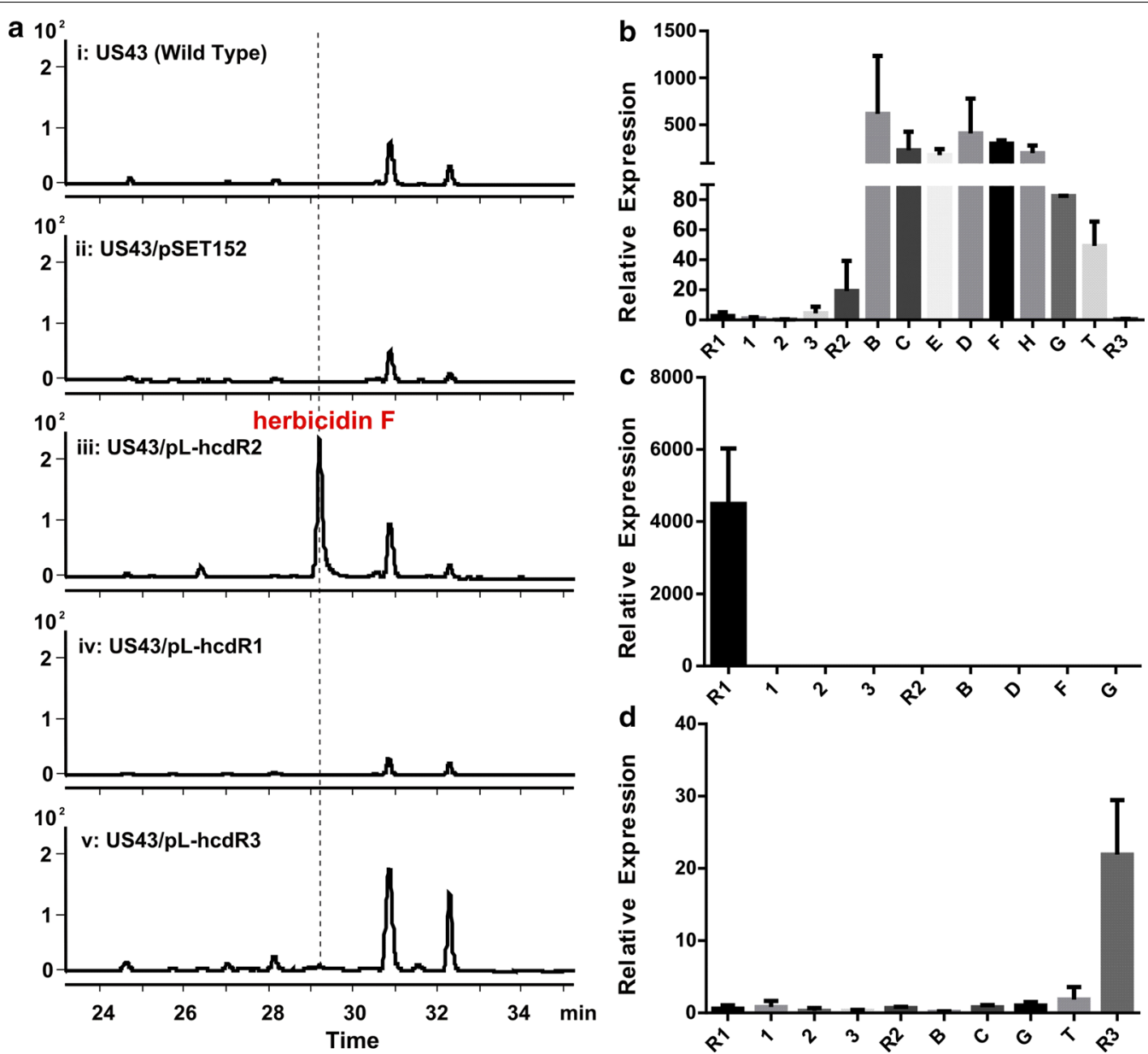

Fig. 2 Effects of overexpression of possible regulators on herbicidin production and gene expression. a Analysis of the production of herbicidin F in the fermentation broth of different strains by HPLC. i, S. mobaraensis US-43; ii, US43/pSET152; iii, US43/pL-hcdR2; iv, US43/pL-hcdR1; V, US43/ pL-hcdR3. Transcriptional analysis of different genes in overexpression strains US43/pL-hcdR2 (b), US43/pL-hcdR1 (c) and US43/pL-hcdR3 (d). Data are from three biological samples with two PCR determinations of each. The values were normalized to that of $h r d B$ and were represented as mean \pm SD. The amounts of each particular transcript in the control strain US43/pSET152 were arbitrarily assigned as 1

by MEME-ChIP, a block of MEME Suite. This consensus binding sequence possessed a potential palindromic sequence, consistent with known binding sites of regulators of the LuxR type, such as LuxR [26], TraR [27], LasR [28] and QscR [29]. Then we employed the block FIMO (Find Individual Motif Occurrences) to scan 48 intergenic regions for individual matches to the possible motif matrix. The results showed 30 promoter regions were matched and ranked by $p$ value (Figs. 1,3 ). Each of the 14 scanned strains together with hcd, her and anm cluster possessed at least one 21-bp consensus binding motif with dyad symmetry (Fig. 4b), the majority of which were located in $h c d B$ homologous gene promoter regions. It closely resembled the consensus motifs determined for other LuxR-type regulators, but has a stronger preference for one side of the imperfect palindrome (Fig. 4b, left). These results hinted that orthologues of HcdR2 might regulate the production of herbicidin analogues by binding the consensus DNA sequence in different strains. There are four clusters with the consensus binding sites but without the homologue of HcdR2 nearby, however, in three of them a highly conserved HcdR2 regulator may be found elsewhere in the whole genome. Interestingly, anm also has the consensus binding site at the promoter region of $a n m B$. Although the genome sequence of aureonuclemycin-producing strain (S. aureus var. suzhoueusis) is not available, some genome-mined clusters without herbicidin tailoring genes also have the HcdR2 homologues within their clusters. These results suggest the pathway-specific regulatory mechanism is conserved in different strains harboring $h c d / h b c / h e r / a n m$-like clusters.

To verify whether HcdR2 binds to the predicted DNA sequence, we performed a series of electrophoretic 


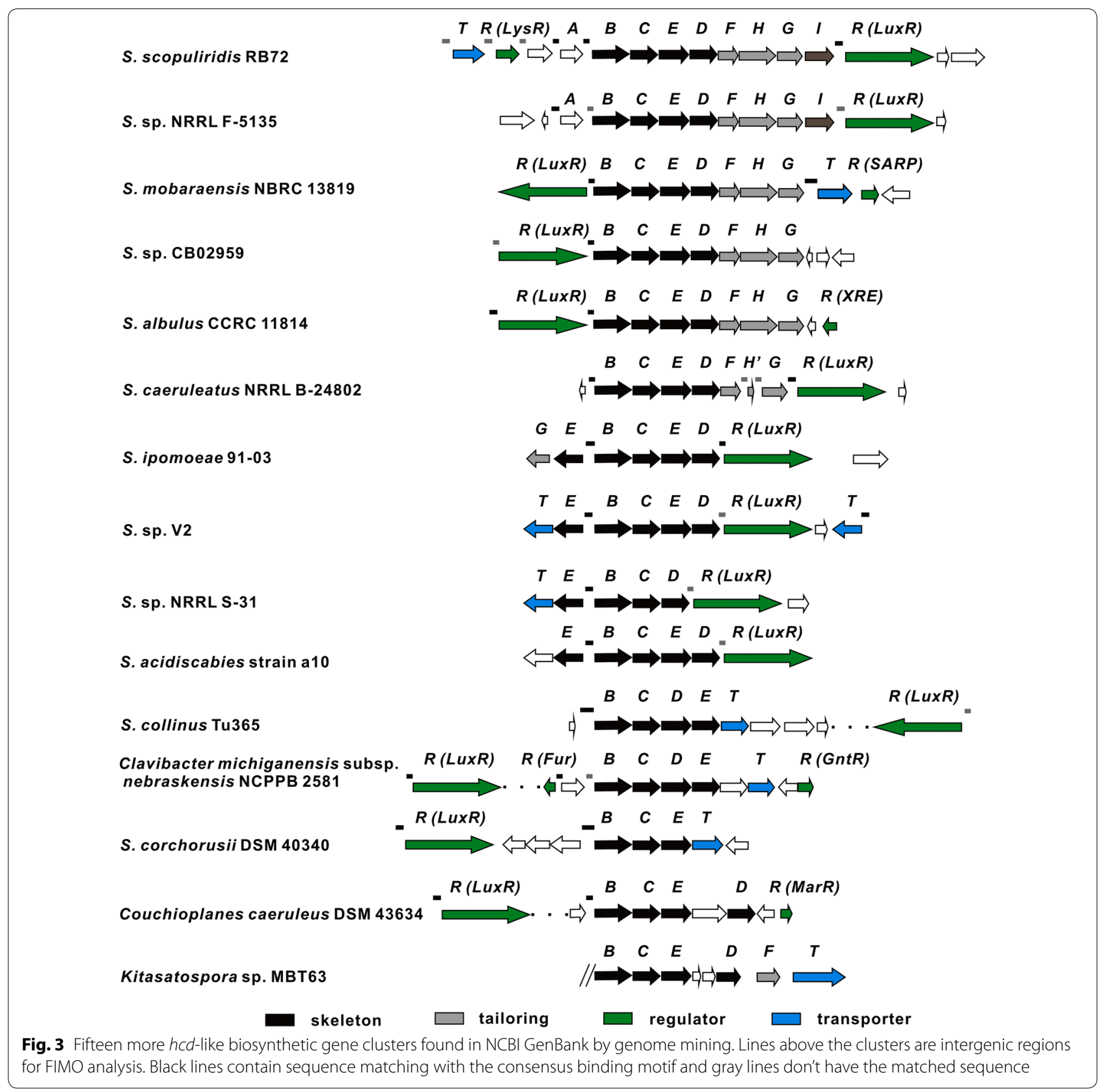

mobility shift assays (EMSAs). HcdR2 was overexpressed in E. coli BL21(DE3) as a $\mathrm{His}_{10}$-tagged protein with a predicted molecular mass of $100,983 \mathrm{Da}$, and purified by nickel affinity chromatography (Additional file 1: Figure S3A). The EMSA results showed that the divergent intergenic region fragment $h c d R 2-B \mathrm{p}$ (containing two consensus binding motifs) and $h c d T$ upstream region fragment $h c d T-2 \mathrm{p}$ (containing a consensus binding motif) were bound by HcdR2 specifically, as shifting of the probes was decreased when the excess unlabeled specific competitor DNA fragments were added into the binding reactions
(Fig. 5b). No shifting of the probe occurred when hcdT$1 \mathrm{p}$ fragment containing no consensus binding motif was analyzed (Additional file 1: Figure S3B). When two unlabeled competitor DNA fragments, her3p from Streptomyces sp. L-9-10 and Bp from Streptomyces sp. V2, were added in excess to binding reactions, shifting of the probes was decreased (Fig. 5c, lanes 2 and 3), which strongly suggested HcdR2 might bind specifically to these two DNA fragments containing a consensus binding motif respectively. Consistent with the motif prediction, an excess of $h c d R 1 \mathrm{p}$ containing no consensus binding 


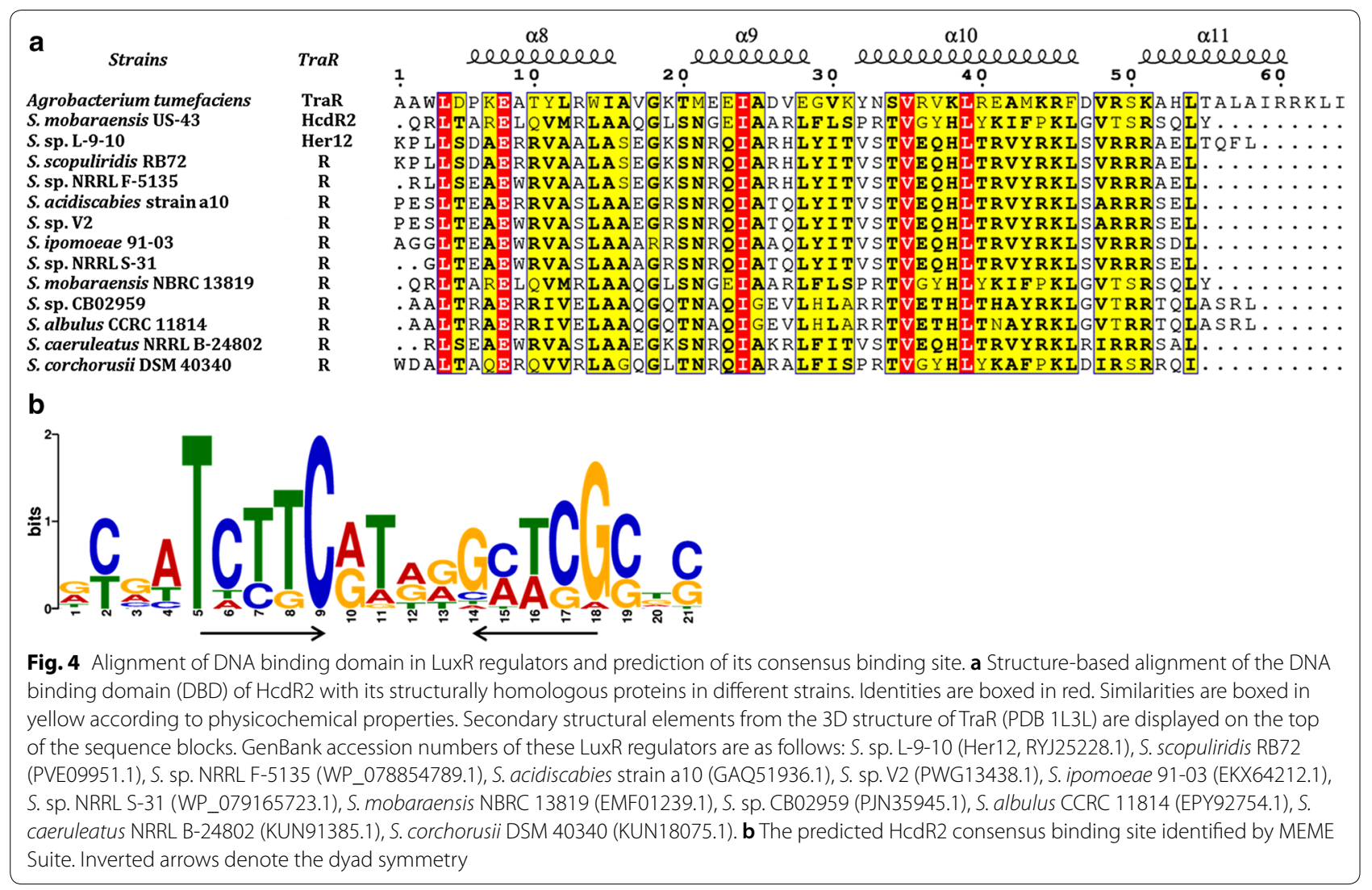

motif didn't decrease the shifting of the probe (Fig. 5c, lane 4). These results adequately demonstrate that the predicted promoter regions with consensus binding motif (Fig. 3) are regulated by HcdR2. This warranted a reliable strategy to activate newly genome-mined $h c d /$ $\mathrm{hbc/her/anm-like} \mathrm{clusters} \mathrm{by} \mathrm{overexpression} \mathrm{of} \mathrm{HcdR2} \mathrm{or}$ its orthologue in their own cluster.

\section{New herbicidin analogues were discovered from $h c d R 2$ overexpression strain by molecular networking}

With the dramatically increased production of herbicidin F in $h c d R 2$ overexpression strain, some trace herbicidin congeners that were undetectable in the wild type strain were discovered. As molecular networking is a powerful tool to visualize the structurally related molecules [14, 15], we employed it to analyze herbicidin congeners in the fermentation broth of $h c d R 2$ overexpression strain. The crude extract of US43/pLhcdR2 fermentation broth was first analyzed on an Agilent 1200 instrument (Agilent Technologies, Santa Clara, CA, USA) coupled to an LTQ XL ion trap mass spectrometer. The LC-MS/MS data were uploaded to MassIVE server (massive.ucsd.edu) and analyzed using a GNPS based molecular networking workflow to generate molecular networks. The resulting spectral networks were visualized using Cytoscape V3.5.1 [30], where nodes represented precursor masses. A subnetwork containing the node corresponding to herbicidin $\mathrm{F}$ was identified in the whole molecular network from the crude extract of US43/pL-hcdR2 (Fig. 6). This constellation contained ten nodes possessing precursor ions ranging from $\mathrm{m} / z 508$ to 536 . Detail analysis of their LC-ESI(+)MS (Fig. 6) and ESI(+)-MS/MS spectra (Fig. 7a) resulted in identification of four known herbicidins $(\mathbf{1}, \mathbf{2} / \mathbf{3}, \mathbf{4}$, and $\mathbf{5})$ and six potential new herbicidin structures (3/2, 6-10) (Fig. 7b).

Compound 1, herbicidin F, has a molecular weight of 536 and characteristic MS/MS fragmentation patterns (Additional file 1: Figure S2 and Fig. 7). In the ESI(+)MS/MS spectrum of compound 1, fragments $\mathrm{m} / \mathrm{z} 418$ (F3) and 283 (F6) are the main peaks corresponding to $\left[\mathrm{M} \text {-tigly-2 } \mathrm{H}_{2} \mathrm{O}+\mathrm{H}\right]^{+}$and $\left[\mathrm{M} \text {-tigly-adenyl- } 2 \mathrm{H}_{2} \mathrm{O}+\mathrm{H}\right]^{+}$, respectively, and can serve as the diagnostic daughter ions. Fragments $m / z$ 518, 500, 454 (F1), 436 (F2), 319 (F4) and 301 (F5), corresponding to $\left[\mathrm{M}-\mathrm{H}_{2} \mathrm{O}+\mathrm{H}\right]^{+}$, $\left[\mathrm{M}-2 \mathrm{H}_{2} \mathrm{O}+\mathrm{H}\right]^{+}, \quad[\mathrm{M} \text {-tigly }+\mathrm{H}]^{+}, \quad\left[\mathrm{M} \text {-tigly- } \mathrm{H}_{2} \mathrm{O}+\mathrm{H}\right]^{+}$, $[\mathrm{M} \text {-tigly-adenyl }+\mathrm{H}]^{+}$, and $\left[\mathrm{M} \text {-tigly-adenyl- } \mathrm{H}_{2} \mathrm{O}+\mathrm{H}\right]^{+}$, respectively, together with the main fragments $\mathrm{F} 3$ and $\mathrm{F} 6$ constitute the characteristic MS/MS spectral profile of herbicidins. 
a $\begin{array}{lllllllllllllll}R 1 & 1 & 2 & 3 & R 2(L U x R) & B & C & E & D & F & H & G & T & R 3\end{array}$

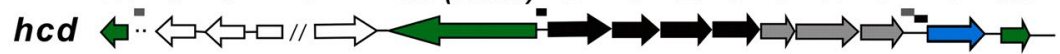
(Streptomyces mobaraensis US43)

her

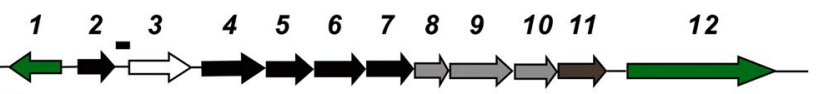

(Streptomyces sp. L-9-10)

hcd-like cluster

(Streptomyces sp. V2)

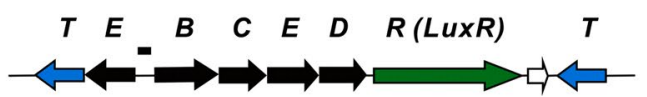

b

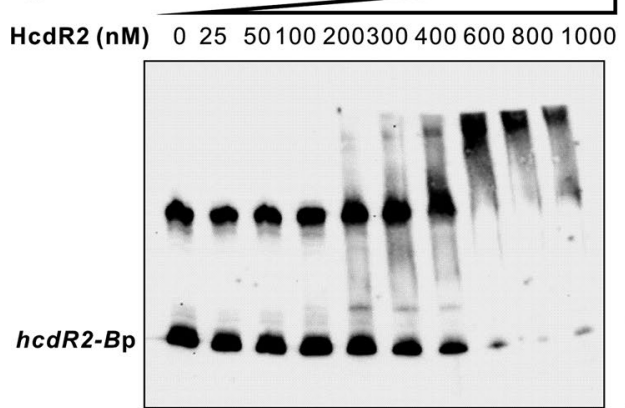

HcdR2 (nM) $0 \quad 255010020040080010002000$

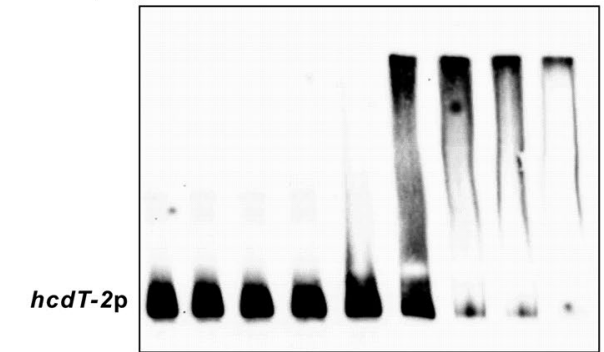

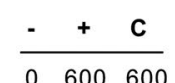
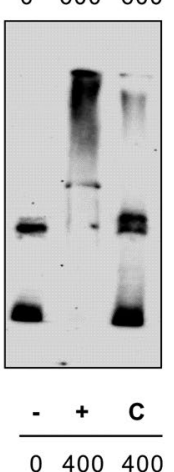

c

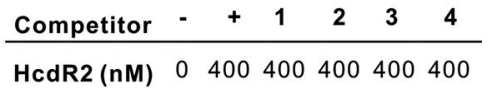

cdR2 (nM) $0 \quad 400400400400400$

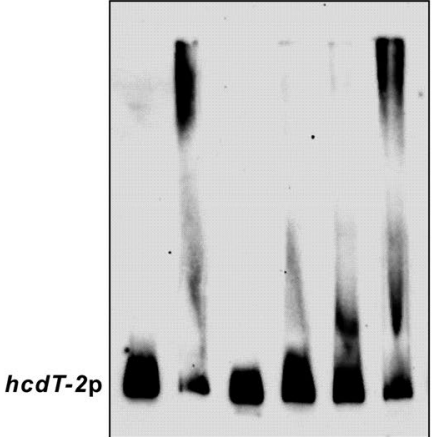

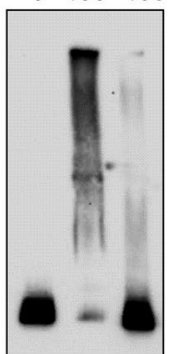

Fig. 5 EMSA analysis of HcdR2 with the postulated promoter regions of the hcd-like clusters. a Three hcd-like clusters with promoter regions analyzed in EMSA experiments. Black lines above the ORFs are DNA fragments containing HcdR2 consensus binding motif(s), and gray lines are DNA fragments containing no consensus binding motif. $\mathbf{b}$ EMSA assays of $5^{\prime}$ biotin-labeled fragments hcdR2-Bp and hcdT-2p with purified HcdR2. The minus indicates probe only, and the plus indicates probe incubated with $\mathrm{HcdR} 2$ at a certain concentration. $C$ indicates probe incubated a certain concentration of HcdR2 with a 200-fold excess of unlabeled specific competitor DNA fragment. c Competition reactions of 5' biotin-labeled hcdT-2p probe incubated with $400 \mathrm{nM} \mathrm{HcdR2.200-fold} \mathrm{excess} \mathrm{of} \mathrm{unlabeled} \mathrm{competitor} \mathrm{DNA} \mathrm{fragments} \mathrm{were} \mathrm{added} \mathrm{respectively.} \mathrm{1-4} \mathrm{represent} \mathrm{hcdT-2p,}$ her3p from Streptomyces sp. L-9-10, Bp from Streptomyces sp. V2 and hcdR1p respectively

Compounds 2 and 3 showed the similar MS/MS patterns with herbicidin F (1). Furthermore, compound 2 has the same quasi-molecular ion and fragments as compound 3, 14 Da less than those of herbicidin F (1) (Fig. 7), suggestive of the absence of a methyl at the position $R_{2}$ or $R_{3}$. To further characterize their structures, the compounds $2(1 \mathrm{mg})$ and $3(0.5 \mathrm{mg})$ were purified. Both of $\mathbf{2}$ and $\mathbf{3}$ exhibited the characteristic UV spectrum of nucleoside, the maximum absorbance at approximate $260 \mathrm{~nm}$ (Additional file 1: Figure S4). Based on the high resolution electrospray ionization mass spectrometry (HR-ESIMS) $[\mathrm{M}+\mathrm{H}]^{+} \mathrm{m} / z$ 522.1857 (calcd for $\mathrm{C}_{22} \mathrm{H}_{28} \mathrm{O}_{10} \mathrm{~N}_{5}, \quad m / z$ 522.1836), compound $\mathbf{2}$ and $\mathbf{3}$ are determined to have the same molecular formula of $\mathrm{C}_{22} \mathrm{H}_{27} \mathrm{O}_{10} \mathrm{~N}_{5}$, a $\mathrm{CH}_{2}$ less than that of herbicidin $\mathrm{F}$, which further confirmed the above speculation of the absence of methyl at the position $R_{2}$ or $R_{3}$. The position of the methyl was further determined by ${ }^{1} \mathrm{H}$ NMR spectrum. The ${ }^{1} \mathrm{H}$ NMR spectra of compounds 2 and $\mathbf{3}$ were collected in DMSO-d6 to obtain the hydroxyl proton signals which can assist to assign the position of methyl. In DMSO-d6, both compounds had two comparable sets of ${ }^{1} \mathrm{H}$ NMR signals (appr. 1:0.7 for 2 and 2:3 for 3, Additional file 1: Figures S5, S6). This phenomenon arose from the equilibrium between hemiketal and free carbonyl forms 

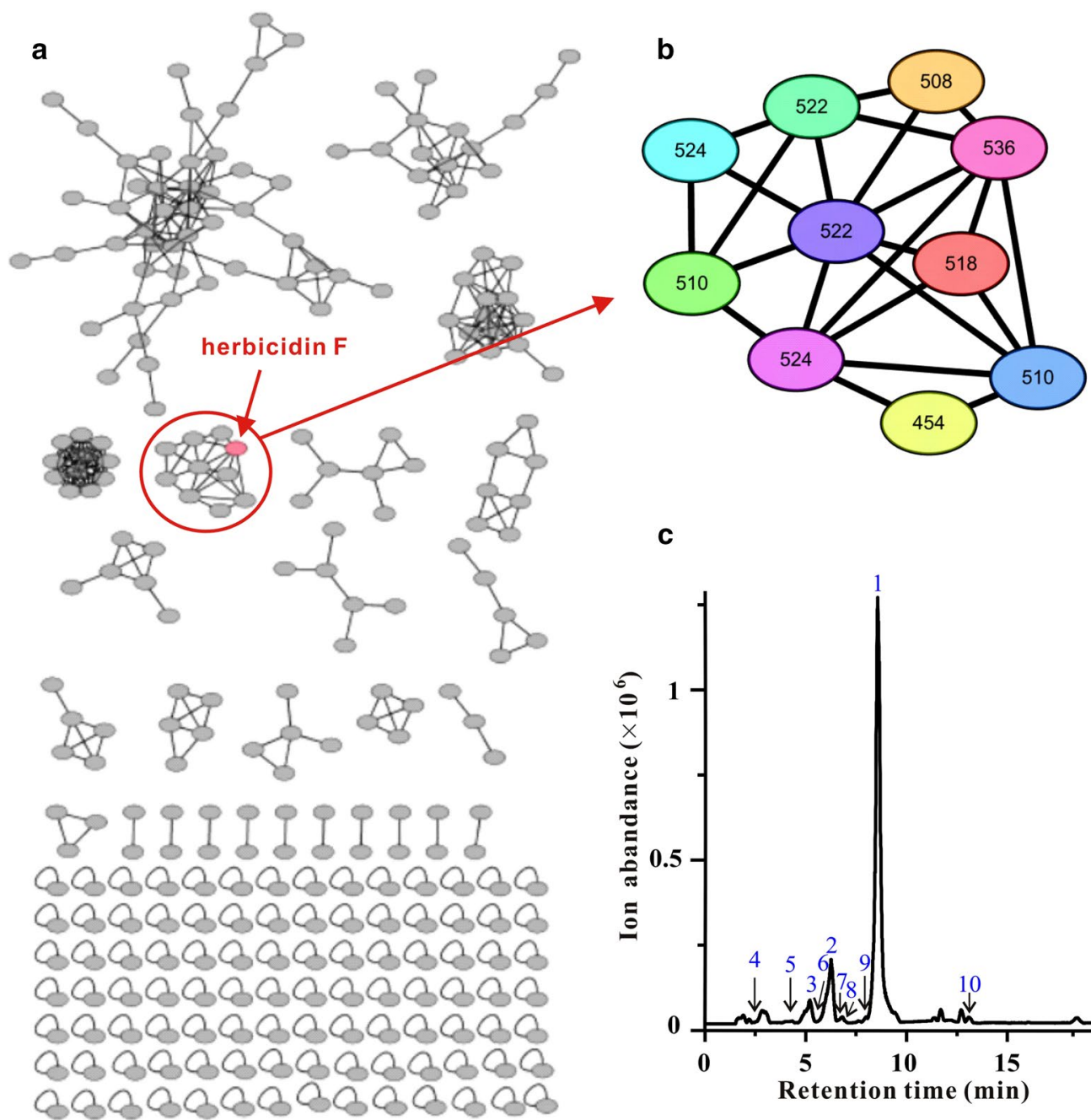

C

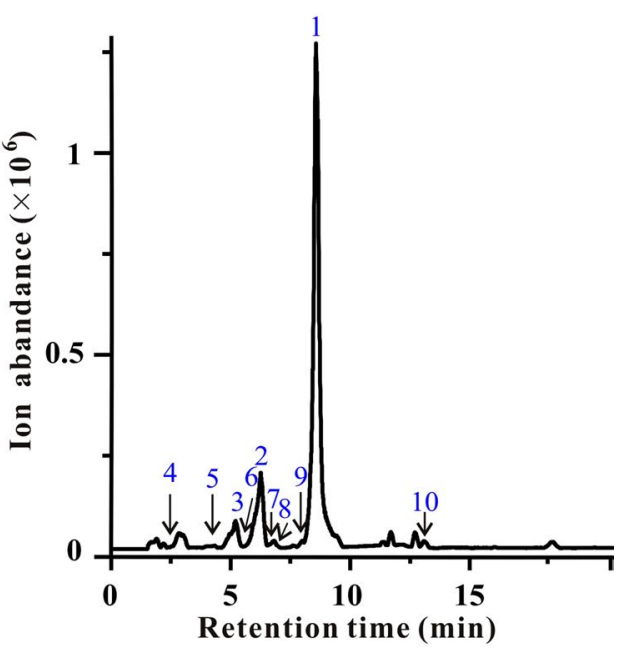

Fig. 6 Molecular networking directed discovery of new herbicidin analogues. a Molecular network consisting of all parent ions detected by LC-MS in the extract crude of $h c d R 2$ overexpression strain. $\mathbf{b}$ A constellation for potential herbicidins was picked out using herbicidin $\mathrm{F}$ as a probe and amplified for displaying. This constellation has ten nodes possessing precursor ions ranging from $\mathrm{m} / \mathrm{z} 508$ to $536[\mathrm{M}+\mathrm{H}]^{+}$(Node labels show the precursor masses). c Based on the molecular networking results, the ten herbicidin peaks (1-10) corresponding to the ten nodes were found in the LC-MS spectrum

of herbicidins, which had also been observed for $11^{\prime}$-O-demethylherbicidin A and 11'-O-demythylherbicidin $\mathrm{B}$ in $\mathrm{D}_{2} \mathrm{O}$ according to the literature [13]. To be convenient for comparison, the solvent was switched to DMSO- $d_{6}$ for compound 1. Comparing the ${ }^{1} \mathrm{H}$ NMR spectrum of $\mathbf{2}$ with that of $\mathbf{1}$ (Fig. 8) revealed the absence of $\mathrm{H}-2^{\prime}-\mathrm{OCH}_{3}$ signal $(\delta 3.32(\mathrm{~s}, 3 \mathrm{H}) / 3.34(\mathrm{~s}$, $3 \mathrm{H})$ ) in the former, which confirmed that $\mathbf{2}$ is short of a methyl at the position $\mathrm{R}_{2}$ and has the same structure as herbicidin $\mathrm{K}$. Comparing the ${ }^{1} \mathrm{H}$ NMR spectrum of 3 with those of $\mathbf{1}$ and $\mathbf{2}$ (Fig. 8) indicated the loss of $\mathrm{H}-11^{\prime}-\mathrm{OCH}_{3}$ signal $(\delta 3.50(\mathrm{~s}, 3 \mathrm{H}) / 3.67(\mathrm{~s}, 3 \mathrm{H}))$ and the presence of $\mathrm{H}-11^{\prime}-\mathrm{COOH}$ signal $(\delta 13.00(\mathrm{~s}, 1 \mathrm{H})$ in the former, which confirmed that $\mathbf{3}$ is short of a methyl at the position $\mathrm{R}_{3}$, thus a new herbicidin $\mathrm{F}$ analogue bearing a free carboxyl group at $\mathrm{C}-11^{\prime}$, which was named as herbicidin $\mathrm{O}$.

The total or partial structures of compounds 4-10 were tentatively deduced by comparison of their MS/MS fragments with those of 1-3 (Fig. 7). Compounds 4 and 5 were determined to be herbicidin $\mathrm{G}$ and $\mathrm{B}$, according to the quasi-molecular ions (28 $\mathrm{Da}$ and $82 \mathrm{Da}$ less than that of $\mathbf{1}$, respectively) and diagnostic fragments. Compounds 6 and 7 exhibited the same F3 ([M-tigly- $\left.2 \mathrm{H}_{2} \mathrm{O}+\mathrm{H}\right]^{+}$, $m / z 404)$ and F6 ([M-tigly-adenyl- $\left.2 \mathrm{H}_{2} \mathrm{O}+\mathrm{H}\right]^{+}, m / z$ 269) fragments as compounds 2 and $\mathbf{3}$, suggesting that compounds 6 and 7 differ from 2 and 3 in $R_{1}$ substituents. The quasi-molecular ion of $7[\mathrm{M}+\mathrm{H}]^{+}$at $m / z 524$ was 


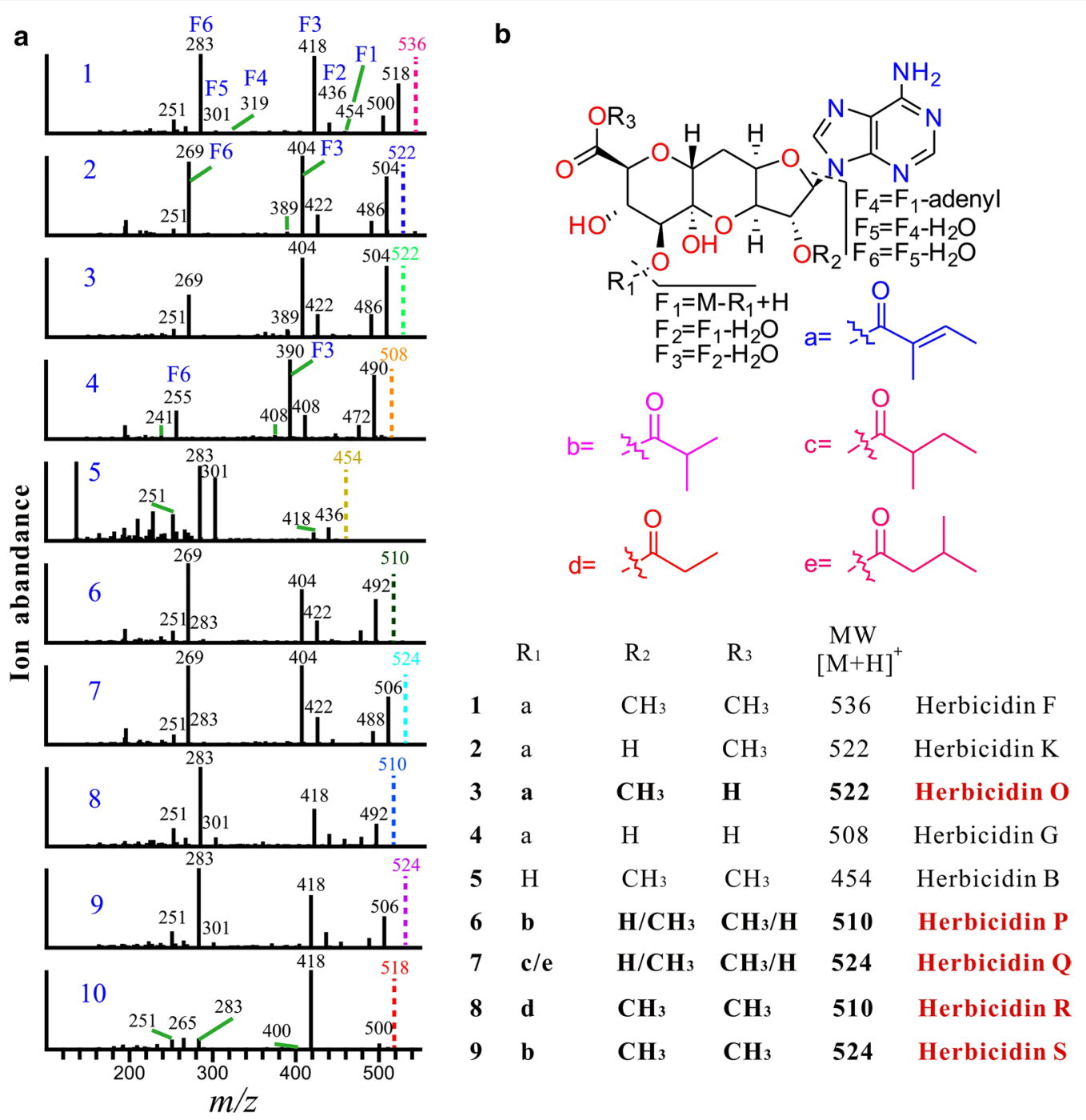

Fig. 7 ESI(+)-MS/MS data and structures of ten herbicidin analogues. a MS/MS analysis for compounds $\mathbf{1 - 1 0}$. The parent ions are indicated in dotted line and the same color as that of the corresponding nodes in GNPS. The diagnostic fragments are indicated. $\mathbf{b}$ The tentative structures of compounds 1-9. The potential new herbicidin analogues are highlighted in red color

2 Da more than those of 2 and 3, indictive of the reduction of the double bond in tigly group and the probable substitute of tigly group in $\mathbf{2}$ with isovaleryl or 2-methylbutyryl group in 7 . The molecular weight of $\mathbf{6}$ showed $14 \mathrm{Da}$ less than that of 7 , suggestive of the shortage of a methyl group and the presence of isobutyryl group at $R_{1}$ of $\mathbf{6}$, the same as the substituent of herbicidin E. Due to the small amounts of compounds obtained, the position of methyl at $\mathrm{R}_{2}$ or $\mathrm{R}_{3}$ in $\mathbf{6}$ and $\mathbf{7}$ are not determined. Compounds 8 and 9 have the same F3 ([M-tigly- $\left.2 \mathrm{H}_{2} \mathrm{O}+\mathrm{H}\right]^{+}$, $m / z 418)$ and F6 ([M-tigly-adenyl- $\left.2 \mathrm{H}_{2} \mathrm{O}+\mathrm{H}\right]^{+}, m / z$ 283) fragments as herbicidin $F(\mathbf{1})$, indictive of the only difference between them in $R_{1}$ substituents. The molecular mass of 8 showed 26 Da less than that of herbicidin F, suggesting that $\mathbf{8}$ should possess a propionyl group at $\mathrm{R}_{1}$. The quasi-molecular ion of $9[\mathrm{M}+\mathrm{H}]^{+}$at $m / z 524,12 \mathrm{Da}$ less than that of herbicidin F, indicating that 9 might possess an isobutyryl group at $R_{1}$, the same as the substituents of herbicidin $\mathrm{E}$ and compound $\mathbf{6}$. Compound $\mathbf{1 0}$ exhibited molecule ion $[\mathrm{M}+\mathrm{H}]^{+}$at $m / z 518,18$ Da less than herbicidin $\mathrm{F}$, and the same diagnostic fragments $\mathrm{F} 3$ ([M-tigly- $\left.\left.\mathrm{H}_{2} \mathrm{O}+\mathrm{H}\right]^{+}, m / z 418\right)$ and $\mathrm{F} 6$ ([M-tigly-adenyl$\left.\mathrm{H}_{2} \mathrm{O}+\mathrm{H}\right]^{+}, m / z$ 283) as herbicidin $\mathrm{F}(\mathbf{1})$, indicative of the loss of $\mathrm{H}_{2} \mathrm{O}$ molecule in compound 10. Although the structures of these congeners can't be determined undoubtedly due to the trace amounts of compounds, the combination of molecular networking and manual analysis of MS/MS gave a valuable clue to the diversity of 


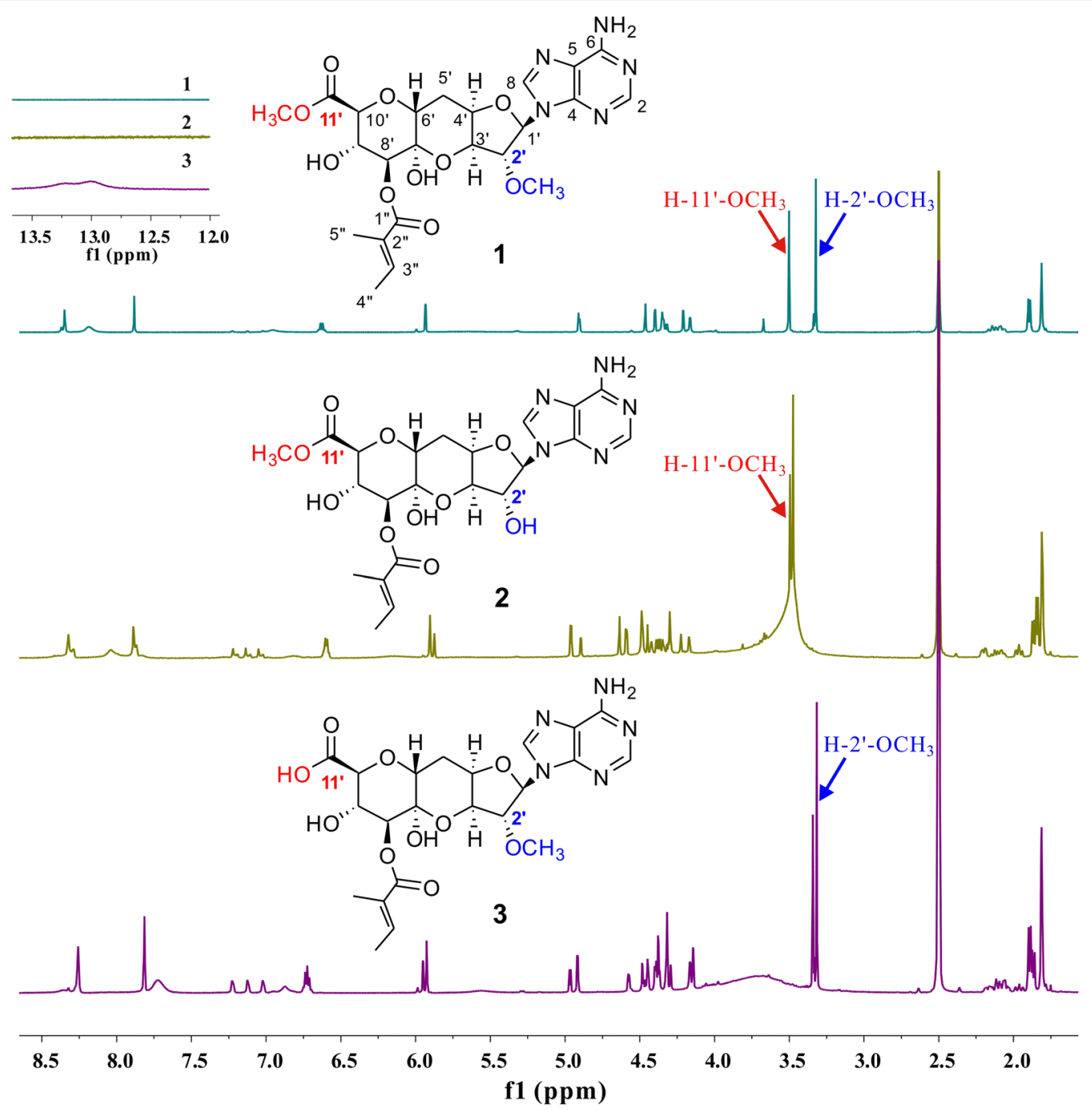

Fig. 8 The comparison of ${ }^{1} \mathrm{H}$ NMR spectra for 1,2 and 3 (in DMSO- $d_{6}$ )

herbicidin variants. Compounds $\mathbf{1 - 1 0}$ are mainly diversified in $\mathrm{R}_{1}$ substituents, which can be tigly, propionyl, isobutyryl, and 2-methylbutyrl/isovaleryl.

\section{Discussion}

In this work, a herbicidin biosynthetic gene cluster $(h c d)$ was identified in $S$. mobaraensis US- 43 by bioinformatics analysis. The seven structural genes are homologous to the two reported clusters her and $h b c$. In these clusters, multiple regulators were present in each cluster (Fig. 1b), which brings a question which one is the pathwayspecific regulator for herbicidin biosynthesis. HcdR2, belonging to LuxR family, was identified as the positive pathway-specific regulator by its overexpression and then improvement of the production level of herbicidin F by about 20-fold, which makes it easier to isolate and identify herbicidin $\mathrm{F}$ and its congeners. What's more, 15 more $h c d / h b c / h e r / a n m$-like clusters were found in NCBI GenBank by genome mining, most of which contained one LuxR-type regulator situated in the cluster. These regulators showed similarities in 3D structure, especially in the C-terminal DNA binding domain and N-terminal AAA ATPase domain. As expected, a consensus binding sequence of HcdR2 was detected in the intergenic regions in all of the clusters by bioinformatics analysis, at least one in each cluster. Although this 21-bp consensus motif exhibits dyad symmetry, HcdR2 showed a unique characteristic with the less conserved sequence on the right side of palindrome, which probably results from the structural differences in the HcdR2-like proteins (Fig. 4b). Furthermore, the EMSA results confirmed that the promoter regions containing the consensus binding motif were regulated by HcdR2 or its orthologues. Therefore, we speculated these HcdR2-like regulators are conserved in hcd/ 
$\mathrm{hbc/her/anm-like} \mathrm{clusters} \mathrm{and} \mathrm{play} \mathrm{a} \mathrm{positive} \mathrm{role} \mathrm{in} \mathrm{the}$ biosynthesis of herbicidin/aureonuclemycin congeners by binding consensus DNA sequence, which providing a strategy for activating novel $h c d / h b c / h e r / a n m-l i k e ~ c l u s-$ ters to discover and identify more herbicidin/aureonuclemycin analogues.

The transcription analysis of predicted genes showed that HcdB-H and HcdT are responsible for the biosynthesis of herbicidin F. Compared with her and $h b c$, transporter is unique in $h c d$ and responsible for herbicidin transportation. The seven structural genes are homologous to both her4 10 [13] and $h b c B \sim H$ [12]. According to the recently reported characterization of the biosynthetic pathway for herbicidins, we speculated that the biosynthesis of herbicidin $\mathrm{F}$ was firstly catalyzed by $\mathrm{HcdB} / \mathrm{C} / \mathrm{D} / \mathrm{E}$ for core assembly, then the serine hydrolase $(\mathrm{HcdH})$ for tiglyl loading and last two steps of SAMdependent methylation (HcdF/G). Because of the lack of $h b c I / h e r 11$ encoding a cytochrome P450 monooxygenase in $h c d$, which catalyzed the hydroxylation reaction on tiglyl moiety, no compounds have been found with hydroxylation on acyl group $\left(\mathrm{R}_{1}\right)$ in $S$. mobaraensis US-43 so far. Unlike our original prediction, none of Hcd $1 / 2 / 3$ was related to the biosynthesis of the tiglyl moiety to the core of the herbicidins according to the transcriptional analysis. Recently, Lin et al. [13] speculated that the biosynthesis of the tiglyl moiety follows a pathway similar to what is observed in plant, which might be also present in $S$. mobaraensis US-43. A recent report by Tang's group reported that $\mathrm{HbcH}$ catalyzed the transfer of tiglyl-CoA to form herbicidins by in vivo disruption and in vitro enzymatic assays [12]. Furthermore, the substrate spectrum of acyltransferase $\mathrm{HbcH}$ was also investigated in vitro and many acyl groups can be transferred to form a series of derivatives in their study [12]. Here, the six newly identified herbicidin congeners in fermentation broth of HcdR2 overexpressed S. mobaraensis US-43 were diverse in acyl groups including propionyl, isobutyryl and 2-methylbutyryl/isovaleryl, which was consistent with their in vitro result and indicated that the substrate flexibility of the serine hydrolase $(\mathrm{HcdH})$ was a useful feature for generating new herbicidin analogues.

A 21-bp consensus binding sequence of HcdR2 was detected using the on-line program MEME Suite. The results showed 30 promoter regions matched and each of the 17 scanned strains possessed at least one consensus binding site (Figs. 1, 3). These clusters can be divided into 2 groups. One only contains genes for the bare tricyclic core assembly similar to anm and the other group has additional tailoring genes similar to $h c d$. In our cluster, there are two predicted binding sites situated between $h c d R 2$ and $h c d B$ and one in $h c d T$ promoter region respectively (Fig. 1), which were confirmed to be bound by HcdR2 through a series of EMSAs. No binding site was discovered in $h c d R 1, h c d R 3, h c d 1$ and $h c d 2$ promoter regions, which was consistent with the transcription analysis of $\mathrm{HcdR} 1 \sim 3$ in overexpression strains. All of the promoter regions of $h c d B$ and its homologue occupied a binding site except in the clusters from $S$. sp. NRRL F-5135 and Clavibacter michiganensis subsp. nebraskensis NCPPB 2581. In these two clusters, there is a consensus binding site existing in the promoter region of the upstream gene in the same direction as in clusters of S. sp. L-9-10 and S. scopuliridis RB72. Except for the upstream gene in Clavibacter michiganensis subsp. nebraskensis NCPPB 2581, the other three upstream genes are homologous to $h b c A$. HbcA was originally thought to catalyze the esterification of $-\mathrm{OH}$ at $\mathrm{C}-8^{\prime}$, but it was confirmed not involved in this reaction later [12]. Here the promoter region of her3 (homologous to $h b c A$ ) was found to be bound by HcdR2 (Fig. 5c, lane 2), which hint $\mathrm{HbcA} / \mathrm{Her} 3$ may be somehow related to the biosynthesis of herbicidin analogues which have not be identified. In addition, there were some binding sites located in the promoter regions of HcdR2-like regulators, suggesting this regulator may control the expression of itself, possibly involved in the feedback regulation of the herbicidin production. Several transporter genes also had this consensus binding site in their promoter region. Among them, transporters in anm-like clusters have similarity with AnmT and belong to MFS superfamily, which may be conserved in anm cluster. Besides, a few of new genes were present in the genome-mined clusters and the prediction of the binding sites showed they could express along with other genes, indicating that many novel analogues with more diversity are yet to be discovered. This will be useful for identification and characterization of new biosynthetic parts or modules for herbicidins/aureonuclemycin analogues and lay a foundation for the applications of synthetic biology.

Here, based on the dramatical improvement of the expression of herbicidin gene cluster, we employed molecular networking to analyze the secondary metabolites of $h c d R 2$ overexpression strain. As a result, herbicidin $\mathrm{F}$ and nine other compounds formed a subcluster in the network, and then six new herbicidin congeners were identified by MS/MS spectral analysis. Among them, several congeners were trace components and hard to be distinguished by manual, while they can be easily picked out by automatic molecular networking. In addition, the MS/MS data of herbicidin F in this research has been uploaded to the GNPS library, which will assist GNPS users to find herbicidin congeners from crude extract even if there are no references of herbicidins at hand. Nowadays, with the number of microbial genome sequences growing 
rapidly, much more $h c d / h b c / h e r / a n m$-like clusters might be discovered by genome mining. Combining with molecular networking, the overexpression of HcdR2 or its orthologue will facilitate the exploitation of novel herbicidins.

\section{Conclusions}

In this study, a herbicidin biosynthetic gene cluster (hcd) was identified in S. mobaraensis US-43, a strain known for production of bleomycin analogues. Among three potential regulators, HcdR2, belonging to LuxR family, was identified as the conserved, positive pathway-specific regulator for herbicidin biosynthesis by overexpression and then the analysis of production level of herbicidin $\mathrm{F}$ as well as transcription analysis of the cluster. The homologues of HcdR2 are present in most of the genome-mined hcd/hbc/her/anm-like clusters. What's more, at least one 21-bp consensus binding motif of HcdR2 was identified in each cluster, suggesting HcdR2 is conserved for herbicidin/aureonuclemycin production. Combined with molecular networking, ten herbicidin congeners were picked out from the secondary metabolites of $h c d R 2$ overexpression strain, six new herbicidin analogues were identified by MS/MS spectral analysis, and the structure of herbicidin $\mathrm{O}$ was further confirmed by ${ }^{1} \mathrm{H}$ NMR spectrum. These results indicated that the combination of $h c d R 2$ overexpression and molecular networking is an effective way to activate cryptic $h c d$-like clusters discovered by genome mining, and lay a foundation for the identification of novel herbicidins.

\section{Materials and methods}

Strains, plasmids and growth conditions

The wild-type $S$. mobaraensis US-43 and its derivatives used in this study are listed in Table 2 . The wild-type $S$. mobaraensis US-43, isolated from the soil of Pingyang, Zhejiang, China, was used as a host strain for the propagation and transformation. S. mobaraensis US-43 and its derivatives were grown at $28{ }^{\circ} \mathrm{C}$ on solid S5 medium [31] for sporulation, on mannitol soya flour (MS) agar medium [32] for conjugation and in the liquid phage medium [33] for isolation of genomic DNA. Herbicidin F was produced with two stage liquid state fermentation. The liquid seed fermentation medium $(0.3 \%$ high nitrogen corn starch powder, $2 \%$ soybean powder, $2.5 \%$ glucose, $2 \%$ starch, $2 \%$ maltose, $0.2 \% \mathrm{~K}_{2} \mathrm{HPO}_{4}$, and $0.3 \%$ $\mathrm{NaCl}$ ) and fermentation medium (the same as seed medium) were used in the first and secondary fermentation. Escherichia coli DH5 $\alpha$ [34] was used as a host for general cloning experiments. E. coli ET12567/pUZ8002 [35] was used for conjugal transfer according to the established protocol [32]. E. coli strains were incubated in Luria-Bertani medium (LB) [34] at $37{ }^{\circ} \mathrm{C}$. When required, strains were incubated with apramycin (Am, $50 \mu \mathrm{g} / \mathrm{mL}$ ), ampicillin (Amp, $100 \mu \mathrm{g} / \mathrm{mL}$ ), kanamycin $(\mathrm{Km}, 50 \mu \mathrm{g} / \mathrm{mL})$ and chloramphenicol $(\mathrm{Cm}, 25 \mu \mathrm{g} / \mathrm{mL})$.

\section{Construction of $h c d R 1, h c d R 2$ and $h c d R 3$ gene overexpression strains}

For overexpression of $h c d R 1$ in S. mobaraensis US-43, the complete $h c d R 1$ gene was amplified using the primer pair pL-hcdR1-F/pL-hcdR1-R in Additional file 1: Table S3. And the PCR product of the $h c d R 1$ gene was cloned into

Table 2 Strains and plasmids used in this study

\begin{tabular}{|c|c|c|}
\hline Strains/plasmids & Relevant characteristics & References \\
\hline \multicolumn{3}{|l|}{ Strains } \\
\hline Streptomyces mobaraensis US-43 & Wild-type strain (herbicidin F-producing strain) & Our laboratory \\
\hline US-43/pL-hcdR1 & S. mobaraensis US-43 with the expression vector pL-hcdR1, Am ${ }^{r}$ & This work \\
\hline US-43/pL-hcdR2 & S. mobaraensis US-43 with the expression vector pL-hcdR2, Am ${ }^{r}$ & This work \\
\hline US-43/pL-hcdR3 & S. mobaraensis US-43 with the expression vector pL-hcdR3, Am ${ }^{r}$ & This work \\
\hline \multicolumn{3}{|l|}{ Escherichia coli } \\
\hline $\mathrm{DH} 5 \mathrm{a}$ & General cloning host & {$[34]$} \\
\hline ET12567/pUZ8002 & Donor strain for intergeneric conjugation between E. coli and Streptomyces, $\mathrm{Cm}^{\mathrm{r}}, \mathrm{Km}^{\mathrm{r}}$ & {$[35]$} \\
\hline \multicolumn{3}{|l|}{ Plasmids } \\
\hline pSET152 & Streptomyces integrative vector, $\mathrm{Am}^{r}$ & {$[20]$} \\
\hline pL646 & pSET152 derivative containing the constitutive promoter ermEp* ${ }^{*} \mathrm{Am}^{r}$ & {$[21]$} \\
\hline pL-hcdR1 & pL646 derivative plasmid containing 684 bp complete coding region of $h c d R 1, \mathrm{Am}^{\mathrm{r}}$ & This work \\
\hline pL-hcdR2 & pL646 derivative plasmid containing 2793 bp complete coding region of $h c d R 2, \mathrm{Am}^{r}$ & This work \\
\hline pL-hcdR3 & pL646 derivative plasmid containing 786 bp complete coding region of hcdR3, Amr & This work \\
\hline pET16b-HcdR2 & pET16b derivative plasmid containing 2793 bp complete coding region of hcdR2, Amp ${ }^{r}$ & This work \\
\hline
\end{tabular}


the NdeI-BamHI sites of pL646 [21], a pSET152 [20] -derived expression plasmid with a strong constitutive promoter ermEp* in the upstream of the multiple cloning sites. With the same strategy, the $h c d R 2$ and $h c d R 3$ gene were cloned into the NdeI-BamHI and NdeI-XbaI sites, respectively. The resulted recombinant plasmid pL-hcdR1, pL-hcdR2 and pL-hcdR3 were introduced into E. coli $\mathrm{ET} 12567 / \mathrm{pUZ} 8002$ and then transferred into S. mobaraensis US-43 by conjugation respectively. The plasmid pSET152 [20] was transferred to S. mobaraensis US-43 as controls.

\section{Analysis of herbicidin $\mathrm{F}$ production}

Streptomyces mobaraensis US-43 wild type and the mutants were cultured on solid S5 medium at $28{ }^{\circ} \mathrm{C}$ for 7 days. The spores of $S$. mobaraensis US- 43 and the mutants were inoculated in $100 \mathrm{~mL}$ seed culture and incubated at $28{ }^{\circ} \mathrm{C}$ for $48 \mathrm{~h}$ at $220 \mathrm{rpm}$. Then $5 \mathrm{~mL}$ of the resulting culture was seeded into $100 \mathrm{~mL}$ of the fermentation medium. This production culture was incubated at $28{ }^{\circ} \mathrm{C}$ at $220 \mathrm{rpm}$ for 7 days. The obtained supernatants were analyzed for the production of herbicidin $\mathrm{F}$ by LC-MS. For analyzing the analogues, the supernatant of fermentation broth was enriched by Sep-Pak $C_{18}$ Classic Cartridge (Waters Associates), eluted with 50\% and $100 \%$ methanol solution. HPLC was performed using a C18 column (Agilent, $150 \mathrm{~mm} \times 4.6 \mathrm{~mm}, 5 \mu \mathrm{m}$ ) with UV detection at $210 \mathrm{~nm}$ and $254 \mathrm{~nm}$ on an Agilent 1100 instrument (Agilent Technologies, Santa Clara, CA, USA). The samples were eluted with mobile phase $\mathrm{CH}_{3} \mathrm{OH}-\mathrm{H}_{2} \mathrm{O}$ using a flow rate of $1 \mathrm{~mL} / \mathrm{min}$ : 0-5 min, 5\% $\mathrm{CH}_{3} \mathrm{OH}$; 5-45 min, 5-100\% $\mathrm{CH}_{3} \mathrm{OH}$; 45-55 min, $100 \% \mathrm{CH}_{3} \mathrm{OH} ; 56-60 \mathrm{~min}, 5 \% \mathrm{CH}_{3} \mathrm{OH}$.

\section{Transcriptional analysis by real-time RT-PCR (qRT-PCR)}

Mycelia of S. mobaraensis US-43 grown in fermentation medium for $48 \mathrm{~h}$ were collected and frozen in liquid nitrogen. RNA was extracted using the TRIzol reagent according to the protocol (Promega), and treated with DNaseI to remove any contaminating chromosomal DNA. The quantity and purity of the harvested RNA was determined using a NanoDrop 8000 spectrophotometer (Thermo Scientific). $2 \mu \mathrm{g}$ of each of the total RNA was used as a template for reverse transcription (RT), which was carried out using the TransScript ${ }^{\circledR}$ One-Step gDNA Removal and cDNA Synthesis SuperMix (Transgen). Gene fragments were amplified from the target genes and detected using the Real-Time PCR Detection System (Bio-Rad). The gene primers used in qRT-PCR reactions are listed in Additional file 1: Table S3. Each reaction mixture was comprised of $12.5 \mu \mathrm{L}$ of FastStart Universal $\mathrm{SYBR}^{\circledR}$ Green Master (ROX) (Roche), $2.5 \mu \mathrm{L}$ of template,
$2.5 \mu \mathrm{L}$ of forward primer, $2.5 \mu \mathrm{L}$ of reverse primer and 5 $\mu \mathrm{L}$ of RNase-free $\mathrm{H}_{2} \mathrm{O}$.

\section{Bioinformatics analysis}

The draft genome of $S$. mobaraensis US-43 was sequenced on a second-generation sequencing platform, Illumina Hiseq 2000, resulting in $1204 \mathrm{Mb}$ data $(9,902,314$ reads with $463 \mathrm{bp}$ average insert size and about 150 -fold average coverage). The genome was assembled into 49 scaffolds, 169 contigs $(7,899,533$ bp with a GC content of $73.11 \%$ ) by SOAPdenovo v2.04 [36]. Secondary metabolite biosynthesis gene clusters were predicted by antiSMASH 5.0.0 (Additional file 1: Table S1) [37, 38]. BLASTP was used for genome mining of potential herbicidin/aureonuclemycin clusters using $h c d B / C / D / E$ as targets. Every gene in each cluster was blasted and annotated. HHpred and BLASTP were used to analyze the $3 \mathrm{D}$ structure and conserved domains. The intergenic regions in each cluster were picked out and submitted to the MEME Suite sever (http://meme-suite.org, motifbased sequence analysis tools) for MEME-ChIP analysis. The locations of the discovered sequence with the highest score in each cluster were collected and submitted for MEME analysis to gain a motif. For further verification of the discovered motif, FIMO analysis was carried out to scan a set of intergenic regions for individual matches to this motif. The $p$-value of a motif occurrence is defined as the probability of a random sequence of the same length as the motif matching that position of the sequence with as a good or better score and it was set to less than 0.001 .

\section{Expression and purification of $\mathrm{His}_{10}$-tagged HcdR2 $^{-}$}

The coding region of $h c d R 2$ was amplified from $S$. mobaraensis US-43 genomic DNA with primers HcdR216b-F2 and HcdR2-16b-R2 (Additional file 1: Table S3), then cloned into pET-16b vector (Novagen) between $N d e \mathrm{I}$ and BamHI sites, generating the recombinant plasmid pET16b-HcdR2. Then it was transformed into E. coli BL21(DE3) for protein expression after authenticated by sequencing. E. coli BL21(DE3)/pET16b-HcdR2 were grown in $400 \mathrm{~mL} \mathrm{LB}$ medium with $100 \mu \mathrm{g} / \mathrm{mL}$ ampicillin at $37{ }^{\circ} \mathrm{C}$ to exponential growth phase $\left(\mathrm{OD}_{600}\right.$ of 0.7$)$. IPTG was then added (final concentration $1 \mathrm{mM}$ ), and the cultures were incubated at $15{ }^{\circ} \mathrm{C}$ for $24 \mathrm{~h}$. The cells were harvested by centrifugation $\left(4000 \times g, 10 \mathrm{~min}, 4^{\circ} \mathrm{C}\right)$, washed twice with binding buffer $\left(20 \mathrm{mM} \mathrm{Na}{ }_{3} \mathrm{PO}_{4}\right.$, $500 \mathrm{mM} \mathrm{NaCl}, 20 \mathrm{mM}$ imidazole, $\mathrm{pH}$ 7.4), resuspended in $30 \mathrm{~mL}$ of the same buffer and lysed by sonication on ice. Cellular debris was removed by centrifugation $\left(12,000 \times g, 20 \mathrm{~min}, 4{ }^{\circ} \mathrm{C}\right) . \mathrm{His}_{10}$-tagged HcdR2 was then purified using HisTrap ${ }^{\mathrm{TM}}$ HP (GE Healthcare) according to the manufacturer's instructions, and eluted with elution buffer $\left(20 \mathrm{mM} \mathrm{Na}_{3} \mathrm{PO}_{4}, 500 \mathrm{mM} \mathrm{NaCl}, 500 \mathrm{mM}\right.$ 
imidazole, $\mathrm{pH}$ 7.4) using linear gradient. Fractions eluted from the column with $160 \mathrm{mM}$ imidazole were dialyzed against TGEK buffer $(50 \mathrm{mM}$ Tris base, $10 \%$ glycerol, $1 \mathrm{mM}$ EDTA, $100 \mathrm{mM} \mathrm{KCl}, \mathrm{pH} 8.0)$ at $4{ }^{\circ} \mathrm{C}$ by PD-10 desalting column (GE Healthcare) according to the manufacturer's instructions, and then stored at $-80{ }^{\circ} \mathrm{C}$. Protein purity was determined by Coomassie Brilliant Blue staining after SDS-PAGE on $8 \%$ polyacrylamide gel. The concentration of the purified HcdR2 was determined using Pierce BCA Protein Assay Kit (Thermo Scientific).

\section{Electrophoretic mobility shift assays (EMSAs)}

Promoter fragments were generated by PCR using the primers labeled at their $5^{\prime}$-ends with Biotin (Additional file 1: Table S3) and used as probes in EMSAs. Each $20 \mu \mathrm{L}$ binding reaction consisted of $2 \mu \mathrm{L} 10 \times$ binding buffer (100 mM Tris-HCl, $500 \mathrm{mM} \mathrm{KCl,} 10 \mathrm{mM} \mathrm{DTT}$, pH 7.5), $20 \mathrm{fmol}$ labeled probe and $25-2000 \mathrm{nM}$ of purified protein. In competition reactions, different unlabeled probes ( $>200$ fold of the labeled probes) were added respectively. Reactions were incubated at room temperature for $20 \mathrm{~min}$ and then run on ative 5\% (80:1) acrylamide: bis-acrylamide gel, buffered in $0.5 \times \mathrm{TBE}$ at $120 \mathrm{~V}, 4{ }^{\circ} \mathrm{C}$. The gel was then transferred to nylon membrane (Amersham Biosciences) by electrophoretic transfer. The biotin end-labeled DNA was detected by LightShift Chemiluminescent EMSA Kit (Thermo Scientific) according to the manufacturer's instructions.

Global natural product social molecular networking (GNPS) To acquire the LC-MS/MS data for GNPS analysis, the fermentation broth of US43/pL-hcdR2 was enriched using macroporous absorbent resin 4006 column and eluted by $30 \%$ and $80 \%$ acetone aqueous, respectively. The eluent of $80 \%$ acetone was concentrated under pressure, and then was fractioned by flash ODS column. The fractions containing herbicidins were combined to yield the crude extract. Then the crude extract was analyzed on an Agilent 1200 instrument (Agilent Technologies, Santa Clara, CA, USA) coupled to an LTQ XL ion trap mass spectrometer (Thermo Fisher Scientific, Waltham, MA, USA), using a VP-ODS column $(150 \mathrm{~mm} \times 4.6 \mathrm{~mm}$, $5 \mu \mathrm{m}$, SHIMADZU), with a $1 \mathrm{~mL} / \mathrm{min}, 60 \mathrm{~min}$ gradient elution (the same as above). LC-ESI(+)MS/MS data, acquired at a collision energy of $35 \mathrm{eV}$ as .raw file format, were converted to .mzXML file format using MS convert program of ProteoWizard 3.0 and uploaded to MassIVE server (massive.ucsd.edu). The data are analyzed using GPNS molecular networking tool following the instruction provided in the website of https:/gnps.ucsd.edu/ ProteoSAFe/static/gnps-splash2.jsp. The resulting spectral networks are visualized using Cytoscape version 3.5.1 [30], where nodes represented precursor mass.

\section{Purification and characterization of compound 1-3}

Strain US43/pL-hcdR2 fermentations were scaled up for separation and purification of new analogues. The mycelia were removed by centrifugation, and the supernatant (ca. $4 \mathrm{~L}$ ) was loaded on a column of macroporous absorbent resin $4006(400 \mathrm{~mL})$, and after washing with water, the active absorbed materials were eluted with a step gradient elution (30\%, $80 \%$ and $100 \%$ acetone in water) to give three fractions, Fr1 to Fr3. Based on the HPLC analysis results, herbicidin $\mathrm{F}$ and their derivatives were found in Fr $2(1.197 \mathrm{~g})$. Fr2 was separated by reversed-phase flash column chromatography (RediSep column: $40 \mathrm{~g} \mathrm{C}_{18}$ ) eluting with $14.8 \%$ acetonitrile aqueous containing $0.01 \%$ TFA as modifier to afford five subfractions (Fr2-1 to Fr25). Fr2-3 (262 mg) was purified by semipreparative HPLC (ReproSil-Pur Basic-C18 column, $5 \mu \mathrm{m}, 250 \times 10 \mu \mathrm{mm}$ ) eluting with $18 \%$ acetonitrile aqueous containing $0.01 \%$ TFA as modifier to yield compound $\mathbf{1}(11 \mathrm{mg}), \mathbf{2}(1 \mathrm{mg}), \mathbf{3}$ $(0.5 \mathrm{mg})$. The samples were analyzed on SHIMADZU LC20A instrument using ReproSil-Pur Basic-C18 column $(5 \mu \mathrm{m}, 150 \times 4.6 \mathrm{~mm})$, the same eluent as semipreparative HPLC, and the detector at $254 \mathrm{~nm}$. The HRESIMS data were acquired on Waters ${ }^{\circledR}$ UPLC equipped with $\mathrm{Xevo}^{\circledR}$ G2-S QTof. The NMR data were acquired with Bruker spectrometers using DMSO- $d_{6}$ or $\mathrm{CD}_{3} \mathrm{OD}$ as solvent.

${ }^{1} H$ and ${ }^{13} \mathrm{C}$ NMR spectroscopic characterization of $1{ }^{1} \mathrm{H}$ NMR $\left(600 \mathrm{MHz}, \mathrm{CD}_{3} \mathrm{OD}\right) \delta 8.36(\mathrm{~s}, 1 \mathrm{H}, \mathrm{H}-2)$, 8.09 (s, $1 \mathrm{H}, \mathrm{H}-8), 6.71\left(\mathrm{q}, \mathrm{J}=7.1 \mathrm{~Hz}, 1 \mathrm{H}, \mathrm{H}-3^{\prime \prime}\right), 6.09(\mathrm{~d}$, $\left.\mathrm{J}=1.6 \mathrm{~Hz}, 1 \mathrm{H}, \mathrm{H}-1^{\prime}\right), 5.00\left(\mathrm{~d}, \mathrm{~J}=3.2 \mathrm{~Hz}, 1 \mathrm{H}, \mathrm{H}-8^{\prime}\right), 4.52$ $\left(\mathrm{dd}, \mathrm{J}=10.3,5.7,1 \mathrm{H}, \mathrm{H}-6^{\prime}\right)$ and $4.50(\mathrm{~d}, \mathrm{~J}=1.8 \mathrm{~Hz}, 1 \mathrm{H}$, H-3'), 4.45 (s, $\left.1 \mathrm{H}, \mathrm{H}-10^{\prime}\right), 4.41$ (q, J=2.4 Hz, 1H, H-4'), $4.30\left(\mathrm{dd}, \mathrm{J}=3.2,1.0 \mathrm{~Hz}, 1 \mathrm{H}, \mathrm{H}-9^{\prime}\right), 4.08(\mathrm{~d}, \mathrm{~J}=1.1 \mathrm{~Hz}$, $\left.1 \mathrm{H}, \mathrm{H}-2^{\prime}\right), 3.61\left(\mathrm{~s}, 3 \mathrm{H}, \mathrm{H}-11^{\prime}-\mathrm{OCH}_{3}\right), 3.41$ (s, 3H, H-2'$\left.\mathrm{OCH}_{3}\right), 2.30-2.22\left(\mathrm{~m}, 2 \mathrm{H}, \mathrm{H}-5^{\prime}\right), 1.90(\mathrm{~d}, \mathrm{~J}=7.0 \mathrm{~Hz}, 3 \mathrm{H}$, $\left.\mathrm{H}-4^{\prime \prime}\right), 1.85$ (s, 3H, H-5"). ${ }^{13} \mathrm{C}$ NMR (150 MHz, CD $\left.\mathrm{OD}\right)$

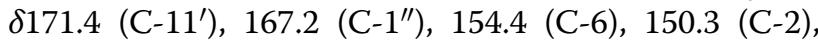
149.5 (C-4), $142.1\left(\mathrm{C}-3^{\prime \prime}\right), 141.8(\mathrm{C}-8), 128.6\left(\mathrm{C}-2^{\prime \prime}\right), 119.9$ (C-5), $93.5\left(\mathrm{C}-7^{\prime}\right), 91.8\left(\mathrm{C}-2^{\prime}\right), 89.1\left(\mathrm{C}-1^{\prime}\right), 79.4\left(\mathrm{C}-4^{\prime}\right), 78.4$ (C-10'), $74.7\left(\mathrm{C}-3^{\prime}\right), 72.0\left(\mathrm{C}-8^{\prime}\right), 70.6\left(\mathrm{C}-9^{\prime}\right), 66.7\left(\mathrm{C}-6^{\prime}\right)$, $58.5\left(\mathrm{C}-2^{\prime}-\mathrm{OCH}_{3}\right), 52.8\left(\mathrm{C}-11^{\prime}-\mathrm{OCH}_{3}\right), 26.7\left(\mathrm{C}-5^{\prime}\right), 15.2$ $\left(\mathrm{C}-4^{\prime \prime}\right), 12.5\left(\mathrm{C}-5^{\prime \prime}\right)$.

${ }^{1} H$ NMR spectroscopic characterization of $\mathbf{1}$ (mixture of hemiacetal and free carbonyl forms) ${ }^{1} \mathrm{H}$ NMR (500 MHz, DMSO) $\delta 8.37(\mathrm{~s}, 1 \mathrm{H}, \mathrm{H}-2) /, 8.35(\mathrm{~s}, 1 \mathrm{H}, \mathrm{H}-2)$, $8.19\left(\mathrm{~s}, 2 \mathrm{H}, \mathrm{H}-\mathrm{NH}_{2}\right) 7.88(\mathrm{~s}, 1 \mathrm{H}, \mathrm{H}-8), 6.63(\mathrm{qd}, J=6.9$, $\left.1.2 \mathrm{~Hz}, 1 \mathrm{H}, \mathrm{H}-3^{\prime \prime}\right), 5.93\left(\mathrm{~d}, J=1.9 \mathrm{~Hz}, 1 \mathrm{H}, \mathrm{H}-1^{\prime}\right) / 6.00(\mathrm{~d}$, $\left.J=1.6 \mathrm{~Hz}, 1 \mathrm{H}, \mathrm{H}-1^{\prime}\right), 4.91\left(\mathrm{~d}, J=3.1 \mathrm{~Hz}, 1 \mathrm{H}, \mathrm{H}-8^{\prime}\right), 4.46$ (s, $\left.1 \mathrm{H}, \mathrm{H}-10^{\prime}\right), 4.40\left(\mathrm{~d}, J=2.2 \mathrm{~Hz}, 1 \mathrm{H}, \mathrm{H}-3^{\prime}\right), 4.33(\mathrm{dd}$, $\left.J=11.6,5.4 \mathrm{~Hz}, 1 \mathrm{H}, \mathrm{H}-6^{\prime}\right), 4.21\left(\mathrm{~d}, J=1.8 \mathrm{~Hz}, 1 \mathrm{H}, \mathrm{H}-4^{\prime}\right)$, 4.17 (s, 1H, H-2') 4.16 (dd, J=3.1, $\left.0.9 \mathrm{~Hz}, 1 \mathrm{H}, \mathrm{H}-9^{\prime}\right), 3.50$ $\left(\mathrm{s}, 3 \mathrm{H}, \mathrm{H}-11^{\prime}-\mathrm{OCH}_{3}\right) / 3.67\left(\mathrm{~s}, 3 \mathrm{H}, \mathrm{H}-11^{\prime}-\mathrm{OCH}_{3}\right), 3.32(\mathrm{~s}$, 
$\left.3 \mathrm{H}, \mathrm{H}-2^{\prime}-\mathrm{OCH}_{3}\right) / 3.34\left(\mathrm{~s}, 3 \mathrm{H}, \mathrm{H}-2^{\prime}-\mathrm{OCH}_{3}\right), 2.16-2.05$ (m, $\left.2 \mathrm{H}, \mathrm{H}-5^{\prime}\right), 1.89$ (dd, $\left.J=7.1,1.0 \mathrm{~Hz}, 3 \mathrm{H}, \mathrm{H}-4^{\prime \prime}\right), 1.81(\mathrm{~s}, 3 \mathrm{H}$, $\left.\mathrm{H}-5^{\prime \prime}\right)$.

${ }^{1} H$ NMR spectroscopic characterization of $\mathbf{2}$ (mixture of hemiacetal and free carbonyl forms) ${ }^{1} \mathrm{H}$ NMR $\left(600 \mathrm{MHz}, \mathrm{DMSO}-d_{6}\right) \delta 8.33(\mathrm{~s}, 1 \mathrm{H}, \mathrm{H}-2) / 8.30(\mathrm{~s}, 1 \mathrm{H}$, $\mathrm{H}-2)$, 8.04 (s, $\left.2 \mathrm{H}, \mathrm{H}-\mathrm{NH}_{2}\right) 7.90$ (s, $\left.1 \mathrm{H}, \mathrm{H}-8\right) / 7.88$ (s, $1 \mathrm{H}$, $\mathrm{H}-8), 6.61$ (dd, $\left.J=13.7,6.8 \mathrm{~Hz}, 1 \mathrm{H}, \mathrm{H}-3^{\prime \prime}\right), 5.91(\mathrm{~s}, 1 \mathrm{H}$, $\left.\mathrm{H}-1^{\prime}\right) / 5.88\left(\mathrm{~s}, 1 \mathrm{H}, \mathrm{H}-1^{\prime}\right), 4.97\left(\mathrm{~d}, J=4.1 \mathrm{~Hz}, 1 \mathrm{H}, \mathrm{H}-8^{\prime}\right) / 4.90$ $\left(\mathrm{d}, J=3.1 \mathrm{~Hz}, 1 \mathrm{H}, \mathrm{H}-8^{\prime}\right)$, 4.64-4.18 (m, 6H, H-4', H-6', $\mathrm{H}-10^{\prime}, \mathrm{H}-2^{\prime}, \mathrm{H}-3^{\prime}, \mathrm{H}-9^{\prime}$, signals from hemiacetal form and free carbonyl form overlapped with each other), 3.50 (s, $\left.3 \mathrm{H}, \mathrm{H}-11^{\prime}-\mathrm{OCH}_{3}\right) / 3.48$ (s, $\left.3 \mathrm{H}, \mathrm{H}-11^{\prime}-\mathrm{OCH}_{3}\right), 2.24-$ $1.93\left(\mathrm{~m}, 2 \mathrm{H}, \mathrm{H}-5^{\prime}\right), 1.88\left(\mathrm{~d}, J=7.1 \mathrm{~Hz}, 3 \mathrm{H}, \mathrm{H}-4^{\prime \prime}\right) / 1.85$ (d, $\left.J=7.1 \mathrm{~Hz}, 3 \mathrm{H}, \mathrm{H}-4^{\prime \prime}\right), 1.82\left(\mathrm{~s}, 3 \mathrm{H}, \mathrm{H}-5^{\prime \prime}\right) / 1.81(\mathrm{~s}, 3 \mathrm{H}$, $\left.\mathrm{H}-5^{\prime \prime} \mathrm{H}\right)$.

${ }^{1} H$ NMR spectroscopic characterization of $\mathbf{3}$ (mixture of hemiacetal and free carbonyl forms) ${ }^{1} \mathrm{H}$ NMR $\left(500 \mathrm{MHz}, \mathrm{DMSO}-d_{6}\right) \delta 13.00(\mathrm{~s}, 1 \mathrm{H}, \mathrm{H}-\mathrm{COOH}), 8.26$ (s, 1H, H-2), $7.81(\mathrm{~s}, 1 \mathrm{H}, \mathrm{H}-8), 7.72\left(\mathrm{~s}, 2 \mathrm{H}, \mathrm{H}-\mathrm{NH}_{2}\right), 6.72$ $\left(\mathrm{m}, 1 \mathrm{H}, \mathrm{H}-3^{\prime \prime}\right), 5.95\left(\mathrm{~d}, J=1.9 \mathrm{~Hz}, 1 \mathrm{H}, \mathrm{H}-1^{\prime}\right) / 5.92(\mathrm{~d}$, $\left.J=1.9 \mathrm{~Hz}, 1 \mathrm{H}, \mathrm{H}-1^{\prime}\right), 4.97\left(\mathrm{~d}, J=4.1 \mathrm{~Hz}, 1 \mathrm{H}, \mathrm{H}-8^{\prime}\right) / 4.92$ (d, $\left.J=3.1 \mathrm{~Hz}, 1 \mathrm{H}, \mathrm{H}-8^{\prime}\right), 4.57-4.14\left(\mathrm{~m}, 6 \mathrm{H}, \mathrm{H}-4^{\prime}, \mathrm{H}-6^{\prime}\right.$, $\mathrm{H}-10^{\prime}, \mathrm{H}-2^{\prime}, \mathrm{H}-3^{\prime}, \mathrm{H}-9^{\prime}$, signals from hemiacetal form and free carbonyl form overlapped with each other), 3.34 (s, $\left.3 \mathrm{H}, \mathrm{H}-2^{\prime}-\mathrm{OCH}_{3}\right) / 3.32\left(\mathrm{~s}, 1 \mathrm{H}, \mathrm{H}-2^{\prime}-\mathrm{OCH}_{3}\right), 2.20-1.93$ (m, $\left.2 \mathrm{H}, \mathrm{H}-5^{\prime}\right), 1.89$ (dd, J=7.0, $\left.0.9 \mathrm{~Hz}, 2 \mathrm{H}, \mathrm{H}-4^{\prime \prime}\right) / 1.87$ (dd, $\left.J=7.1,0.8 \mathrm{~Hz}, 2 \mathrm{H}, \mathrm{H}-4^{\prime \prime}\right), 1.81\left(\mathrm{~d}, J=1.0 \mathrm{~Hz}, 3 \mathrm{H}, \mathrm{H}-5^{\prime \prime}\right)$.

\section{Supplementary information}

Supplementary information accompanies this paper at https://doi. org/10.1186/s12934-019-1225-7.

Additional file 1. Additional tables and figures.

\section{Acknowledgements}

We thank Beijing Genomics Institute (Shenzhen, China) for sequencing, assembly and annotation of the genome.

\section{Authors' contributions}

YS and RG performed the experiments, analyzed the primary data and wrote the draft manuscript. YL and XW assisted with the purification of compounds. WR, XL and LW assisted with the construction of overexpression strains and the fermentation. YX supervised the chemical work in this study and revised the manuscript. BH supervised the whole research work and revised the manuscript. All authors read and approved the final manuscript.

\section{Funding}

The Drug Innovation Major Project (2018ZX09711001-007-001 and 2018ZX09711001-006-011), National Natural Science Foundation of China (81872780, 81803410, 81703398, 81630089 and 81621064), CAMS Innovation Fund for Medical Sciences (2016-I2M-3-012, 2016-I2M-2-002, and 2018-I2M-3-005), National Key Research and Development Program of China
(2018YFA0902000) and the Natural Science Foundation of Beijing Municipality (7172137, 7184227).

\section{Availability of data and materials}

The data supporting our findings can be found in the main paper and the additional file.

\section{Ethics approval and consent to participate}

Not applicable.

\section{Consent for publication \\ Not applicable.}

\section{Competing interests}

The authors declare that they have no competing interests.

Received: 14 April 2019 Accepted: 1 October 2019

Published online: 15 October 2019

\section{References}

1. Chen C, Si S, He Q, Xu H, Lu M, Xie Y, Wang Y, Chen R. Isolation and characterization of antibiotic NC0604, a new analogue of bleomycin. J Antibiot (Tokyo). 2008;61:747-51.

2. Ren H, Lu M, Xie YY, Gao N, Xu HZ, Yao TE, He N, He QY, Chen RX. NC1101, a novel tetrahydropyrimidine-containing bleomycin analog from Streptomyces verticillus var. pingyangensis $n$. var. J Antibiot (Tokyo). 2012;65:327-9.

3. Qi X, Wang X, Ren H, Zhang F, Zhang X, He N, Guo W, Chen R, Xie Y, He Q. NC1404, a novel derivative of Bleomycin with modified sugar moiety obtained during the preparation of Boningmycin. J Antibiot (Tokyo). 2017:70:970-3.

4. Arai M, Haneishi T, Kitahara N, Enokita R, Kawakubo K. Herbicidins A and $B$, two new antibiotics with herbicidal activity. I. Producing organism and biological activities. J Antibiot (Tokyo). 1976;29:863-9.

5. Haneishi T, Terahara A, Kayamori H, Yabe J, Arai M. Herbicidins A and B, two new antibiotics with herbicidal activity. II. Fermentation, isolation and physico-chemical characterization. J Antibiot (Tokyo). 1976;29:870-5.

6. Takiguchi Y, Yoshikawa H, Terahara A, Torikata A, Terao M. Herbicidins F and G, two new nucleoside antibiotics. J Antibiot (Tokyo). 1979;32:862-7.

7. Takiguchi Y, Yoshikawa H, Terahara A, Torikata A, Terao M. Herbicidins C and E, two new necleoside antibiotics. J Antibiot (Tokyo). 1979;32:857-61.

8. Chai X, Youn UJ, Sun D, Dai J, Williams P, Kondratyuk TP, Borris RP, Davies J, Villanueva IG, Pezzuto JM, Chang LC. Herbicidin congeners, undecose nucleosides from an organic extract of Streptomyces sp. L-9-10. J Nat Prod. 2014;77:227-33.

9. Ha S, Lee KJ, Lee SI, Gwak HJ, Lee JH, Kim TW, Choi HJ, Jang JY, Choi JS, Kim CJ, et al. Optimization of Herbicidin A Production in Submerged Culture of Streptomyces scopuliridis M40. J Microbiol Biotechnol. 2017;27:947-55.

10. Chen JJ, Rateb ME, Love MS, Xu Z, Yang D, Zhu X, Huang Y, Zhao LX, Jiang Y, Duan Y, et al. Herbicidins from Streptomyces sp. CB01388 showing antiCryptosporidium activity. J Nat Prod. 2018;81:791-7.

11. Tang GL, Li WT, Pan HX, Jian XH: The biosynthetic gene cluster of aureonucleomycin and its application. Chinese Patent, CN201210236348.1. 2012 (in Chinese).

12. Pan HX, Chen Z, Zeng T, Jin WB, Geng Y, Lin GM, Zhao J, Li WT, Xiong Z, Huang SX, et al. Elucidation of the herbicidin tailoring pathway offers insights into its structural diversity. Org Lett. 2019;21:1374.

13. Lin GM, Romo AJ, Liem PH, Chen Z, Liu HW. Identification and interrogation of the herbicidin biosynthetic gene cluster: first insight into the biosynthesis of a rare undecose nucleoside antibiotic. J Am Chem Soc. 2017;139:16450-3.

14. Watrous J, Roach P, Alexandrov T, Heath BS, Yang JY, Kersten RD, van der Voort M, Pogliano K, Gross H, Raaijmakers JM, et al. Mass spectral molecular networking of living microbial colonies. Proc Natl Acad Sci USA. 2012;109:E1743-52.

15. Quinn RA, Nothias LF, Vining O, Meehan M, Esquenazi E, Dorrestein PC. Molecular networking as a drug discovery, drug metabolism, and precision medicine strategy. Trends Pharmacol Sci. 2017;38:143-54. 
16. Caesar LK, Kellogg JJ, Kvalheim OM, Cech RA, Cech NB. Integration of biochemometrics and molecular networking to identify antimicrobials in Angelica keiskei. Planta Med. 2018;84:721-8

17. Jiang ZB, Ren WC, Shi YY, Li XX, Lei X, Fan JH, Zhang C, Gu RJ, Wang $L F$, Xie $Y Y$, Hong B. Structure-based manual screening and automatic networking for systematically exploring sansanmycin analogues using high performance liquid chromatography tandem mass spectroscopy. J Pharm Biomed Anal. 2018;158:94-105.

18. Nothias LF, Nothias-Esposito M, da Silva R, Wang M, Protsyuk I, Zhang Z, Sarvepalli A, Leyssen P, Touboul D, Costa J, et al. Bioactivity-based molecular networking for the discovery of drug leads in natural product bioassay-guided fractionation. J Nat Prod. 2018;81:758-67.

19. Hou XM, Li YY, Shi YW, Fang YW, Chao R, Gu YC, Wang CY, Shao CL. Integrating molecular networking and ${ }^{1} \mathrm{H}$ NMR to target the isolation of chrysogeamides from a library of marine-derived Penicillium fungi. J Org Chem. 2019;84:1228-37.

20. Bierman M, Logan R, O'Brien K, Seno ET, Rao RN, Schoner BE. Plasmid cloning vectors for the conjugal transfer of DNA from Escherichia coli to Streptomyces spp. Gene. 1992;116:43-9.

21. Hong B, Phornphisutthimas S, Tilley E, Baumberg S, McDowall KJ. Streptomycin production by Streptomyces griseus can be modulated by a mechanism not associated with change in the adpA component of the A-factor cascade. Biotechnol Lett. 2007;29:57-64.

22. Robert $X$, Gouet P. Deciphering key features in protein structures with the new ENDscript server. Nucleic Acids Res. 2014;42:W320-4.

23. Bailey $T L$, Elkan C. Fitting a mixture model by expectation maximization to discover motifs in biopolymers. Proc Int Conf Intell Syst Mol Biol. 1994;2:28-36.

24. Grant CE, Bailey TL, Noble WS. FIMO: scanning for occurrences of a given motif. Bioinformatics. 2011;27:1017-8.

25. Machanick P, Bailey TL. MEME-ChIP: motif analysis of large DNA datasets. Bioinformatics. 2011;27:1696-7.

26. Egland KA, Greenberg EP. Quorum sensing in Vibrio fischeri: elements of the lux/ promoter. Mol Microbiol. 1999;31:1197-204.

27. Luo ZQ, Farrand SK. Signal-dependent DNA binding and functional domains of the quorum-sensing activator TraR as identified by repressor activity. Proc Natl Acad Sci USA. 1999;96:9009-14.

28. Gray KM, Passador L, Iglewski BH, Greenberg EP. Interchangeability and specificity of components from the quorum-sensing regulatory systems of Vibrio fischeri and Pseudomonas aeruginosa. J Bacteriol. 1994; 176:3076-80.

29. Lee JH, Lequette Y, Greenberg EP. Activity of purified QscR, a Pseudomonas aeruginosa orphan quorum-sensing transcription factor. Mol Microbiol. 2006;59:602-9.

30. Shannon P, Markiel A, Ozier O, Baliga NS, Wang JT, Ramage D, Amin N, Schwikowski B, Ideker T. Cytoscape: a software environment for integrated models of biomolecular interaction networks. Genome Res. 2003;13:2498-504.

31. Wang $L$, Hu Y, Zhang Y, Wang S, Cui Z, Bao Y, Jiang W, Hong B. Role of sgcR3 in positive regulation of enediyne antibiotic C-1027 production of Streptomyces globisporus C-1027. BMC Microbiol. 2009;9:14.

32. Kieser T, Bibb MJ, Buttner MJ, Chater KF, Hopwood DA. Practical streptomyces genetics. Norwich: John Innes Foundation; 2000.

33. Korn F, Weingartner B, Kutzner HJ. A study of twenty actinophages: morphology, serological relationship and host range. In: Freerksen E, Tarnok I, Thumin H, editors. Genetics of the actinomycetales. New York: Gustav Fisher Verlag; 1978.

34. Sambrook J, Russell DW. Molecular cloning: a laboratory manual. 3rd ed. Cold Spring Harbor Laboratory: Cold Spring Harbor; 2001.

35. Paget MS, Chamberlin L, Atrih A, Foster SJ, Buttner MJ. Evidence that the extracytoplasmic function sigma factor $\sigma^{\mathrm{E}}$ is required for normal cell wall structure in Streptomyces coelicolor A3(2). J Bacteriol. 1999;181:204-11.

36. Li R, Zhu H, Ruan J, Qian W, Fang X, Shi Z, Li Y, Li S, Shan G, Kristiansen K, et al. De novo assembly of human genomes with massively parallel short read sequencing. Genome Res. 2010;20:265-72.

37. Blin K, Wolf T, Chevrette MG, Lu X, Schwalen CJ, Kautsar SA, Suarez Duran HG, de Los Santos ELC, Kim HU, Nave M, et al. antiSMASH 4.0-improvements in chemistry prediction and gene cluster boundary identification. Nucleic Acids Res. 2017;45:W36-41.

38. Blin K, Shaw S, Steinke K, Villebro R, Ziemert N, Lee SY, Medema MH, Weber T. antiSMASH 5.0: updates to the secondary metabolite genome mining pipeline. Nucleic Acids Res. 2019;47:W81-7.

\section{Publisher's Note}

Springer Nature remains neutral with regard to jurisdictional claims in published maps and institutional affiliations.
Ready to submit your research? Choose BMC and benefit from:

- fast, convenient online submission

- thorough peer review by experienced researchers in your field

- rapid publication on acceptance

- support for research data, including large and complex data types

- gold Open Access which fosters wider collaboration and increased citations

- maximum visibility for your research: over $100 \mathrm{M}$ website views per year

At BMC, research is always in progress.

Learn more biomedcentral.com/submissions 